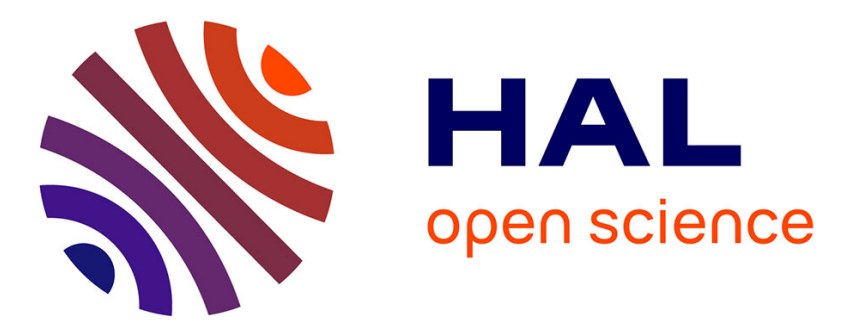

\title{
Coherency Matrix Estimation of Heterogeneous Clutter in High-Resolution Polarimetric SAR Images
}

\author{
Gabriel Vasile, Jean-Philippe Ovarlez, Frédéric Pascal, Céline Tison
}

\section{To cite this version:}

Gabriel Vasile, Jean-Philippe Ovarlez, Frédéric Pascal, Céline Tison. Coherency Matrix Estimation of Heterogeneous Clutter in High-Resolution Polarimetric SAR Images. IEEE Transactions on Geoscience and Remote Sensing, 2010, 48 (4), pp.1809-1826. 10.1109/TGRS.2009.2035496 . hal00466647

\section{HAL Id: hal-00466647 https://hal.science/hal-00466647}

Submitted on 27 Feb 2020

HAL is a multi-disciplinary open access archive for the deposit and dissemination of scientific research documents, whether they are published or not. The documents may come from teaching and research institutions in France or abroad, or from public or private research centers.
L'archive ouverte pluridisciplinaire HAL, est destinée au dépôt et à la diffusion de documents scientifiques de niveau recherche, publiés ou non, émanant des établissements d'enseignement et de recherche français ou étrangers, des laboratoires publics ou privés. 


\title{
Coherency matrix estimation of heterogeneous clutter in high resolution polarimetric SAR images
}

\author{
G. Vasile(1),(4), J. -P. Ovarlez ${ }^{(1),(2)}$, F. Pascal $^{(2)}$ and C. Tison ${ }^{(3)}$ \\ ${ }^{(1)}$ : French Aerospace Lab \\ ONERA DEMR/TSI - Chemin de la Hunière, 91761 Palaiseau Cedex, FRANCE \\ Tel: +33169936313 - Fax: +33169936269 - Email: ovarlez@ onera.fr \\ ${ }^{(2)}$ : Supélec, ONERA, National University of Singapore, Defence Science and Technology Agency Research Alliance \\ SONDRA, Plateau du Moulon, 3 rue Joliot-Curie, 91192 Gif-sur-Yvette Cedex, FRANCE \\ Tel: +33 169851803 - Fax: +33169851809 - Email: frederic.pascal@ supelec.fr \\ ${ }^{(3)}$ : Centre National d'Etudes Spatiales \\ CNES DCT/SI/AR - 18 Av. Edouard Belin, 31401 Toulouse Cedex 9, FRANCE \\ Tel: +33 561282404 - Fax: +33 561283422 - Email: celine.tison@cnes.fr \\ ${ }^{(4)}$ : Grenoble-Image-sPeech-Signal-Automatics Lab, CNRS \\ GIPSA-lab DIS/SIGMAPHY, Grenoble INP - BP 46, 38402 Grenoble Cedex, FRANCE \\ Tel: +33 476826334 - Fax: +33 476574790 - Email : gabriel.vasile@ gipsa-lab.inpg.fr
}

\section{IEEE Transactions on Geoscience and Remote Sensing - Manuscript no. TGRS-2008-00422-R2}

\begin{abstract}
This paper presents an application of the recent advances in the field of Spherically Invariant Random Vectors (SIRV) modelling for coherency matrix estimation in heterogeneous clutter. The complete description of the POLSAR data set is achieved by estimating the span and the normalized coherency independently. The normalized coherency describes the polarimetric diversity, while the span indicates the total received power. The main advantages of the proposed Fixed Point estimator are that it does not require any "a priori" information about the probability density function of the texture (or span) and it can be directly applied on adaptive neighborhoods. Interesting results are obtained when coupling this Fixed Point estimator with an adaptive spatial support based on the scalar span information. Based on the SIRV model, a new maximum likelihood distance measure is introduced for unsupervised POLSAR classification. The proposed method is tested with both simulated POLSAR data and airborne POLSAR images provided by the RAMSES system. Results of entropy/alpha/anisotropy decomposition, followed by unsupervised classification, allow to discuss the use of the normalized coherency and the span as two separate descriptors of POLSAR data sets.
\end{abstract}




\section{CONTENTS}

Nomenclature

I Introduction

II Heterogeneous model for polarimetric textured scenes 5

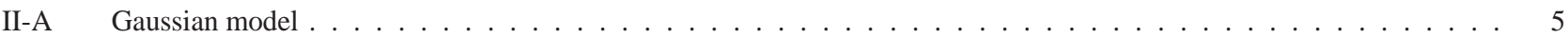

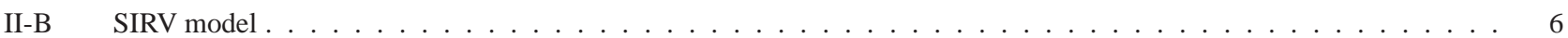

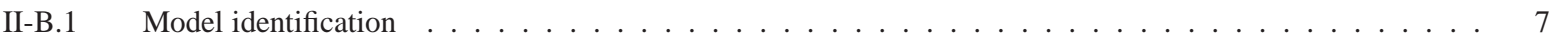

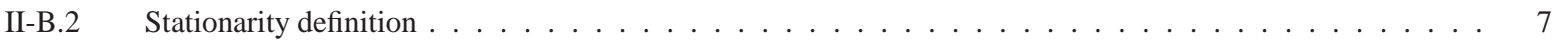

II-B.3 SIRV parameter estimation $\ldots \ldots \ldots \ldots$

II-B.4 Gaussian model in the SIRV context . . . . . . . . . . . . . . . . . . . . . 9

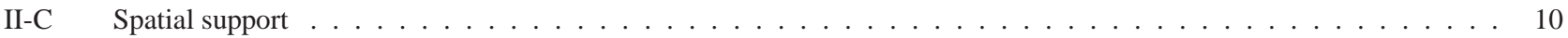

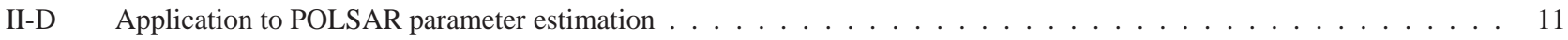

II-E Distance measures for POLSAR segmentation . . . . . . . . . . . . . . . . . . . 12

III Results and discussion 14

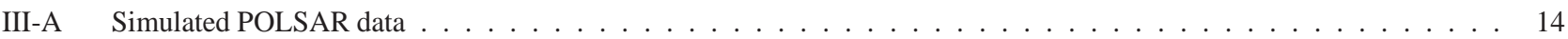

III-A.1 Gaussian case . . . . . . . . . . . . . . . . . . . . . . . . . . . . . . . . 14

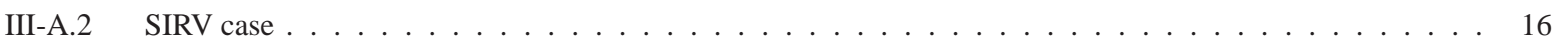

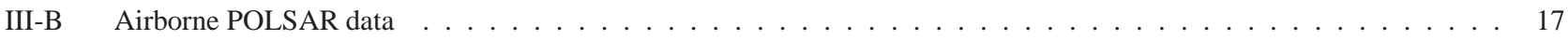

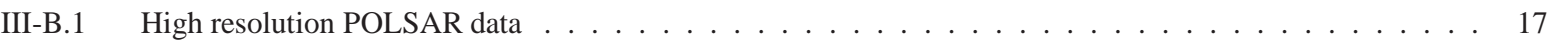

III-B.2 Very high resolution POLSAR data . . . . . . . . . . . . . . . . . . . . . . . . . . . . 19

IV General remarks 20

V Conclusions and perspectives 21

Acknowledgment 22

References 23 
BN : Boxcar Neighborhood.

$[C]$ : generic covariance matrix.

FP : Fixed Point estimator.

i.i.d. : independent and identically distributed.

LLMMSE : Locally Linear Minimum Mean-Squared Error.

$[M]$ : generic normalized polarimetric coherency matrix.

ML : Maximum Likelihood.

MPWF : Multilook Polarimetric Whitening Filter.

$P$ : generic span.

PDF : Probability Density Function.

PWF : Polarimetric Whitening Filter.

SCM : Sample Covariance Matrix.

SDAN : Span Driven Adaptive Neighborhood.

SIRP : Spherically Invariant Random Process.

SIRV : Spherically Invariant Random Vectors.

$[T]$ : generic polarimetric coherency matrix. 


\section{INTRODUCTION}

A Synthetic Aperture Radar (SAR) measures both amplitude and phase of the backscattered signal, producing one complex image for each recording. The sensors being able to emit or receive two orthogonal polarizations, fully POLarimetric Synthetic Aperture Radar (POLSAR) systems describe the interactions between the electromagnetic wave and the target area by means of the Sinclair matrix [1]. Among the difficulties encountered when using POLSAR imagery, one important feature is the presence of speckle. Occurring in all types of coherent imagery, the speckle is due to the random interference of the waves scattered by the elementary targets belonging to one resolution cell [2]. In general, POLSAR data are locally modelled by the multivariate, zero mean, circular Gaussian probability density function, which is completely determined by the covariance matrix [3].

The recently launched POLSAR systems are now capable of producing high quality images of the Earth's surface with meter resolution. The decrease of the resolution cell offers the opportunity to observe much thinner spatial features than the decametric resolution of the up-to-now available SAR images. Recent studies [4] show that the higher scene heterogeneity leads to nonGaussian clutter modelling, especially for urban areas. One commonly used fully polarimetric non-Gaussian clutter model is the product model [5]: the spatial non-homogeneity is incorporated by modelling the clutter as the product between the square root of a scalar random variable (texture) and an independent, zero mean, complex circular Gaussian random vector (speckle). If the texture random variable is supposed to be a Gamma spatial distributed intensity, the product model is equivalent to the well-known $\mathcal{K}$-distributed clutter model [6], [7].

For Gaussian polarimetric clutter model, the estimation of the polarimetric coherency matrix is treated in the context of POLSAR speckle filtering. The POLSAR adaptive filtering techniques can be roughly divided in two main classes [8]: based on the optimization of the spatial support and based on the use of the local statistics to derive adaptive estimators. These two directions are not exclusive since both of them can be applied simultaneously [9], [10]. For example, the refined Lee filter couples eight edge-aligned directional neighborhoods with an adaptive estimator based on the Locally Linear Minimum Mean-Squared Error (LLMMSE) criterion [9].

In the context of non-Gaussian polarimetric clutter models, several studies tackled POLSAR parameter estimation using the product model. For deterministic texture, Novak et al. derived the Polarimetric Whitening Filter (PWF) by optimally combining the elements of the polarimetric covariance matrix to produce a single scalar image [11], [12]. Using the complex Wishart distribution, the PWF for homogeneous surfaces has been generalized to multilook PWF (MPWF) [13], [14]. In general, the texture random variable is specified by the probability density function. For Gamma-distributed texture, Lopès and Séry derived the Maximum Likelihood (ML) estimator of the covariance matrix [13]. Moreover, the vector spatial LLMMSE filter applied on the scalar ML texture estimator has also been introduced, when the texture variance and spatial correlation functions are "a priori" known [13]. In [15], De Grandi et al. performed an extensive study on the dependency of the normalized second-order moment of intensity on polarization state for $\mathcal{K}$-distributed clutter model. This dependency was condensed in graphical form by a formalism called the polarimetric texture signature. This study has been applied for target detection and texture segmentation using the discrete wavelet frame transform generated with the first derivative of a $B$-spline of order three as mother wavelet [16].

The POLSAR information allows the discrimination of different scattering mechanisms. In [17], Cloude and Pottier intro- 
duced the target entropy and the entropy-alpha-anisotropy $(H-\alpha-A)$ model by assigning to each eigenvector the corresponding coherent single scattering mechanism. Based on this decomposition, unsupervised classification for land applications was performed by an iterative algorithm based on complex Wishart density function [18], [19].

The objective of this paper is to present a new coherency estimation technique [20] based on the Spherically Invariant Random Vectors model [21], and to analyze the consequences that this model has on the conventional POLSAR processing chain. The paper is organized as follows. Sect. II is dedicated to the presentation of the proposed estimation scheme. The heterogeneity of polarimetric textured scenes is taken into account by coupling the ML normalized coherency estimator with adaptive neighborhoods driven on the scalar ML span estimators. A new ML distance measure is also introduced for classifying normalized coherency matrices under the SIRV model. In Sect. III, the results obtained using the proposed approach are presented and compared to those given by the Gaussian ML estimator. Results of $H-\alpha-A$ decomposition, followed by unsupervised POLSAR classification allow to discuss the use of the normalized coherency and the span as two separate descriptors of POLSAR data sets. Detailed discussion on the advantages and the limitations of the SIRV model is given in Sect. IV. Eventually, in Sect. V, some conclusions and perspectives are presented.

\section{HETEROGENEOUS MODEL FOR POLARIMETRIC TEXTURED SCENES}

The goal of the estimation process is to derive the scene signature from the observed data set. In the case of spatially changing surfaces ("heterogeneous" or "textured" scenes) the first step is to define an appropriate model describing the dependency between the polarimetric signature and the observable as a function of the speckle. In general, the multiplicative model [5] has been employed for SAR data processing as a product between the square root of a scalar positive quantity (texture) and the description of an equivalent homogeneous surface (speckle) by means of:

- the intensity descriptor for single-polarization SAR images [22], [23],

- the complex SAR signal descriptor for single-polarization SAR data [24],

- the polarimetric target vector descriptor in lexicographic basis for monostatic POLSAR images, [11], [25], [26],

- the normalized polarimetric target vector descriptor in lexicographic basis, [27], [6], [28],

- the polarimetric covariance matrix descriptor for POLSAR data [29], [13].

In this paper, the polarimetric descriptors used are the target vectors $\mathbf{k}=\left[k_{1}, k_{2}, k_{3}\right]^{T}$ in the Pauli basis (monostatic acquisition). The following section presents an application of the recent advances, in the field of Spherically Invariant Random Vectors modelling [20], for estimating span and normalized coherency matrices of high resolution POLSAR data.

\section{A. Gaussian model}

The elements of the vector are generally modelled by a multivariate zero mean, complex, Gaussian random process. The probability density function (PDF) is given by the following expression [2]:

$$
p_{m}(\mathbf{k})=\frac{1}{\pi^{m} \operatorname{det}\{[T]\}} \exp \left\{-\mathbf{k}^{\dagger}[T]^{-1} \mathbf{k}\right\}
$$


where $[T]=E\left\{\mathbf{k k}^{\dagger}\right\}$ is the polarimetric coherency matrix, $\operatorname{det}\{\ldots\}$ denotes the matrix determinant, $\dagger$ is the conjugate transpose operator, $m$ the dimension of the target vector ( $m=3$ for monostatic POLSAR acquisitions) and $E\{\ldots\}$ denotes the statistical mean over the polarimetric channels.

According to Eq. 1, a Gaussian stochastic process is completely characterized by the coherency matrix. In this case, the Maximum Likelihood (ML) estimator of the polarimetric coherency matrix is the Sample Covariance Matrix (SCM) obtain by replacing the statistical mean by spatial averaging:

$$
[\widehat{T}]_{S C M}=\frac{1}{N} \sum_{i=1}^{N} \mathbf{k}_{i} \mathbf{k}_{i}^{\dagger},
$$

where $\mathrm{N}$ is the number of samples. The SCM is statistically determined by the Wishart PDF [2].

Another POLSAR parameter is the span (or total power) $P$ generally defined for each pixel as [1]:

$$
P_{S L C}=\mathbf{k}^{\dagger} \mathbf{k}
$$

The corresponding multilook span can be estimated within a local neighborhood according to:

$$
P=E\left\{\mathbf{k}^{\dagger} \mathbf{k}\right\}=\operatorname{Tr}\{[T]\}
$$

where $\operatorname{Tr}\{[T]\}$ denotes the trace of the matrix $[T]$. Hence, the common span estimator for the Gaussian case can be directly obtained from the SCM as:

$$
\widehat{P}_{S C M}=\operatorname{Tr}\left\{[\widehat{T}]_{S C M}\right\}
$$

\section{B. SIRV model}

Spherically Invariant Random Vectors (SIRV) and their applications to estimation and detection in communication theory were firstly introduced by Kung Yao [21]. The SIRV is a class of non-homogeneous Gaussian processes with random variance. The complex m-dimensional measurement $\mathbf{k}$ is defined as the product between the independent complex circular Gaussian vector $\mathbf{z}$ (speckle) with zero mean and covariance matrix $[M]=E\left\{\mathbf{z z}^{\dagger}\right\}$ and the square root of the positive random variable $\tau$ (representing the texture):

$$
\mathbf{k}=\sqrt{\tau} \mathbf{z}
$$

It is important to notice that in the SIRV definition, the PDF of the texture random variable is not explicitly specified. As a consequence, SIRVs describe a whole class of stochastic processes defined by Eq. 6. This class includes the conventional clutter models having Gaussian, $\mathcal{K}$-distributed, Chi, Rayleigh, Weibull or Rician PDFs [30].

For POLSAR data, the SIRV product model is the product of two separate random processes operating across two different statistical axes:

- The polarimetric diversity is modelled by the multidimensional Gaussian kernel characterized by its covariance matrix $[M]$.

- The randomness of spatial variations in the radar backscattering from cell to cell is characterized by $\tau$. The corresponding random process operates along the spatial axis given by the image support. Relatively to the polarimetric axis, the texture random variable $\tau$ can be viewed as a unknown deterministic parameter from cell to cell. 
One major advantage of the SIRV clutter model is the high degree of generality with respect to other texture-aware models employed in the literature [30], [4]. Nevertheless, this model is founded on the validity of three basic assumptions: the texture random variable affects the backscattered power only, it is multiplicative and spatially uncorrelated. When applied to high resolution POLSAR clutter, the SIRV model postulates that the texture descriptor $\tau$ from Eq. 6 is identical for all polarization channels.

Let now $p(\tau)$ be the texture PDF associated to the SIRV model. The Spherically Invariant Random Process (SIRP) corresponding to Eq. 6 has the following PDF [31]:

$$
F\{p(\tau),[M]\}=p_{m}(\mathbf{k})=\int_{0}^{+\infty} \frac{1}{(\pi \tau)^{m} \operatorname{det}\{[M]\}} \exp \left(-\frac{\mathbf{k}^{\dagger}[M]^{-1} \mathbf{k}}{\tau}\right) p(\tau) \mathrm{d} \tau
$$

1) Model identification: When using the product model, an identification problem can be pointed out: the SIRV model is uniquely defined with respect to the covariance matrix parameter up to a multiplicative constant. Let $\left[M_{1}\right]$ and $\left[M_{2}\right]$ be two covariance matrices such that $\left[M_{1}\right]=\kappa \cdot\left[M_{2}\right],(\forall) \kappa \in \mathbb{R}_{+}^{*}$. Notice that the two sets of parameters defined as $\left\{\tau_{1},\left[M_{1}\right]\right\}$ and $\left\{\tau_{2}=\frac{\tau_{1}}{\sqrt{\kappa}},\left[M_{2}\right]\right\}$ describe the same SIRV. For solving this identification problem, the covariance matrix has to be normalized. In the following the covariance matrix $[M]$ is normalized such that $\operatorname{Tr}\{[M]\}=m$, with $m$ the dimension of the target vector.

One important consequence of the imposed normalization condition is that the resulting normalized polarimetric coherency matrix reveals information concerning the polarimetric diversity only: the total power information is transferred into the texture random variable. The POLSAR data can be fully characterized by coupling the normalized coherency matrix with the span descriptor:

$$
P_{S L C}=\mathbf{k}^{\dagger} \mathbf{k}=\tau\left(\mathbf{z}^{\dagger} \mathbf{z}\right)
$$

When operating on the polarimetric statistical axis, the span for the SIRV case is given by:

$$
P=E\left\{\tau\left(\mathbf{z}^{\dagger} \mathbf{z}\right) ; \tau\right\}=\tau \cdot E\left\{\mathbf{z}^{\dagger} \mathbf{z}\right\}=\tau \cdot \operatorname{Tr}\{[M]\}=\tau \cdot m
$$

An estimate of $P$ can be obtained when considering $\tau$ as an unknown deterministic parameter from cell to cell.

2) Stationarity definition: In the following several generic concepts are recalled. Given a SIRP, this process is wide-sense stationary if and only if both the texture random variable and the speckle random vector are wide-sense stationary. As the speckle is a zero mean complex Gaussian vector, the latter means that the statistical samples $\mathbf{k}_{i}$ used in the estimation process must have the same theoretical covariance matrix $[M]$. This condition is called "matrix stationarity".

However, as the results presented in this section can be applied whatever the texture PDF $(\forall p(\tau))$, the previous properties can be reformulated using the SIRV class of stochastic processes. Given a "matrix stationary" stochastic process, this process is "SIRV homogeneous" if and only if the texture random variable is "texture homogeneous". Where "texture homogeneous" means that it is possible to define a texture PDF $(\exists p(\tau))$ such that the stochastic process can be described by the product model from Eq. 6. We illustrate these properties using four local populations which often occur in practical POLSAR applications:

- One zero mean Gaussian process with covariance matrix $[M]: \mathcal{N}(0,[M])$. Being a "Gaussian stationary 1 " process, it is also "SIRP stationary" and "SIRV homogeneous". This model is widely used for POLSAR data analysis [32].

${ }^{1} \mathrm{~A}$ "Gaussian stationary" process is a stochastic process whose Gaussian PDF does not change when shifted in time or space. 
- Two adjacent Gaussian processes with different covariance matrix: $\mathcal{N}=\left\{\mathcal{N}^{(1)}\left(0,[M]_{1}\right), \mathcal{N}^{(2)}\left(0,[M]_{2}\right)\right\}$. The Gaussian mixture $\mathcal{N}$ is neither "SIRP stationary" nor "SIRV homogeneous" as the "matrix stationarity" condition is not respected. Generally, such cases are treated by employing adaptive estimation schemes [9], [8] in order to approximate the local "Gaussian stationarity" condition.

- One $\mathcal{K}$-distributed process [33] with Gamma distributed texture $p_{G}(\tau ; \bar{\tau}, \nu)$ and covariance matrix $[M]$ : $F_{\mathcal{K}}\left\{p_{G}(\tau ; \bar{\tau}, \nu),[M]\right\}$. This process is "SIRP stationary" as it is " $\mathcal{K}$ stationary ${ }^{2 "}$ " but obviously it is not "Gaussian stationary".

- Two adjacent $\mathcal{K}$-distributed processes with two different Gamma texture $\operatorname{PDF} p_{G}^{(1)}\left(\tau ; \bar{\tau}_{1}, \nu_{1}\right), p_{G}^{(2)}\left(\tau ; \bar{\tau}_{2}, \nu_{2}\right)$ and the same covariance matrix $[M]: F_{\mathcal{K}}=\left\{F_{\mathcal{K}}^{(1)}\left\{p_{G}^{(1)}\left(\tau ; \bar{\tau}_{1}, \nu_{1}\right),[M]\right\}, F_{\mathcal{K}}^{(2)}\left\{p_{G}^{(2)}\left(\tau ; \bar{\tau}_{2}, \nu_{2}\right),[M]\right\}\right\}$. The $\mathcal{K}$-distributed processes $F_{\mathcal{K}}^{(1)}$ and $F_{\mathcal{K}}^{(2)}$ are "SIRP stationary" and " $\mathcal{K}$ stationary", but the mixture $F_{\mathcal{K}}$ is not " $\mathcal{K}$ stationary". Despite this, the process $F_{\mathcal{K}}$ is "SIRV homogeneous" as it is possible to define a texture PDF which models the Gamma mixture. As a consequence, the results presented in this section can still be applied in this case.

In conclusion, the two properties to be verified in order to apply the SIRV model are the "matrix stationarity" and the "texture homogeneity". Moreover, the latter considerably relaxes the "texture stationarity" condition required when using explicit texture models such as the Gamma or the Fisher PDF.

3) SIRV parameter estimation: In the field of target detection for radar applications, the SIRV model led to many investigations [34], [35], [36], [37]. In Eqs. 6 and 7, the normalized covariance matrix is an unknown parameter which can be estimated from ML theory. In [31], Gini et al. derived the exact ML estimate $[\widehat{M}]$ of the normalized covariance matrix when $\tau_{i}$ are assumed to be unknown deterministic parameters. For $N$ independent and identically distributed (i.i.d.) data, the likelihood function to maximize with respect to $[M]$ and $\tau_{i}$, is given by:

$$
L_{\mathbf{k}}\left(\mathbf{k}_{1}, \ldots, \mathbf{k}_{N} ;[M], \tau_{1}, \ldots, \tau_{N}\right)=\frac{1}{\pi^{m N} \operatorname{det}\{[M]\}^{N}} \times \prod_{i=1}^{N} \frac{1}{\tau_{i}^{m}} \exp \left(-\frac{\mathbf{k}_{i}^{\dagger}[M]^{-1} \mathbf{k}_{i}}{\tau_{i}}\right) .
$$

For a given $[\mathrm{M}]$, maximizing $L_{\mathbf{k}}\left(\mathbf{k}_{1}, \ldots, \mathbf{k}_{N} ;[M], \tau_{1}, \ldots, \tau_{N}\right)$ with respect to $\tau_{i}$ yields the texture ML estimator

$$
\widehat{\tau}_{i}=\frac{\mathbf{k}_{i}^{\dagger}[M]^{-1} \mathbf{k}_{i}}{m} .
$$

Replacing $\tau_{i}$ in Eq. 10 by their ML estimates the generalized likelihood is obtained as:

$$
L_{\mathbf{k}}^{\prime}\left(\mathbf{k}_{1}, \ldots, \mathbf{k}_{N} ;[M]\right)=\frac{1}{\pi^{m N} \operatorname{det}\{[M]\}^{N}} \times \prod_{i=1}^{N} \frac{m^{m} \exp (-m)}{\left(\mathbf{k}_{i}^{\dagger}[M]^{-1} \mathbf{k}_{i}\right)^{m}} .
$$

The ML estimator of the normalized covariance matrix in the deterministic texture case is obtained by cancelling the gradient of $L_{\mathbf{k}}^{\prime}$ with respect to $[M]$ as the solution of the following recursive equation:

$$
[\widehat{M}]_{F P}=f\left([\widehat{M}]_{F P}\right)=\frac{m}{N} \sum_{i=1}^{N} \frac{\mathbf{k}_{i} \mathbf{k}_{i}^{\dagger}}{\mathbf{k}_{i}^{\dagger}[\widehat{M}]_{F P}^{-1} \mathbf{k}_{i}}=\frac{m}{N} \sum_{i=1}^{N} \frac{\mathbf{z}_{i} \mathbf{z}_{i}^{\dagger}}{\mathbf{z}_{i}^{\dagger}[\widehat{M}]_{F P}^{-1} \mathbf{z}_{i}} .
$$

This approach has been used in [38] by Conte et al. to derive a recursive algorithm for estimating the matrix $[M]$. This algorithm consists in computing the Fixed Point of $f$ using the sequence $\left([M]_{i}\right)_{i \geq 0}$ defined by:

$$
[M]_{i+1}=f\left([M]_{i}\right) .
$$

${ }^{2} \mathrm{~A}$ " $\mathcal{K}$ stationary" process is a stochastic process whose $\mathcal{K}$ PDF does not change when shifted in time or space. 
This study has been completed by the work of Pascal et al. [20], [39], which recently established the existence and the uniqueness, up to a scalar factor, of the Fixed Point estimator of the normalized covariance matrix, as well as the convergence of the recursive algorithm whatever the initialization. The algorithm can therefore be initialized with the identity matrix $[M]_{0}=$ $\left[I_{m}\right]$. One way to analyze the convergence of the Fixed Point estimator consists in evaluating the following criterion:

$$
C(i)=\frac{\|[\widehat{M}](i+1)-[\widehat{M}](i)\|_{F}}{\|[\widehat{M}](i)\|_{F}},
$$

where $\|\ldots\|_{F}$ represents the Frobenius norm. When computing the FP estimator, Eq. 14 is iterated until $C$ becomes smaller than a predefined lower limit. Note that only few iterations suffice to reach an error less than $10^{-15}$ [20].

It has also been shown in [31] and [38] that the recursive estimation scheme from Eq. 14 can be applied to derive an exact ML estimator of the normalized covariance matrix:

$$
[\widehat{M}]_{M L}=\frac{m}{N} \sum_{i=1}^{N} \frac{h_{m+1}\left(\mathbf{k}_{i}^{\dagger}[\widehat{M}]_{M L}^{-1} \mathbf{k}_{i}\right)}{h_{m}\left(\mathbf{k}_{i}^{\dagger}[\widehat{M}]_{M L}^{-1} \mathbf{k}_{i}\right)} \mathbf{k}_{i} \mathbf{k}_{i}^{\dagger}, \quad \text { with } \quad h_{m}(q)=\int_{0}^{\infty} \tau^{p} \exp \left(\frac{q}{\tau}\right) p(\tau) d \tau .
$$

In the previous equation, the exact ML estimator depends on the texture PDF through the SIRV density generating function $h_{m}(q)$. Chitour and Pascal have been recently demonstrated that Eq. 16 admits a unique solution and that its corresponding iterative algorithm converges to the Fixed Point solution for every admissible initial condition [40]. Pascal et al. have also demonstrated that normalized covariance ML estimator developed under the deterministic texture case (Eq. 13), yields also an approximate ML estimator under stochastic texture hypothesis [20], [39].

We propose to apply these results in estimating normalized coherency matrices for high resolution POLSAR data. The main advantage of this approach is that the local "scene heterogeneity" can be taken into account without any "a priori" hypothesis regarding the texture random variable $\tau$ (Eq. 14 does not depend on $\tau$ ). The obtained Fixed Point is the approximate ML estimate under the stochastic $\tau$ assumption and the exact ML under deterministic $\tau$ assumption. Moreover, the normalized polarimetric coherency matrix estimated using the Fixed Point method is unbiased and asymptotically Gaussian distributed [20], [39].

Note also that the texture estimator from Eq. 11 can be directly linked to the total scattered power (span) according to Eq.9. By estimating the normalized coherency as the Fixed Point solution of Eq. 13, the derived estimate is independent of the total power and it contains polarimetric information only. Using this matrix, it is possible to compute the SIRV span ML estimator for unknown deterministic $\tau$ as:

$$
\widehat{P}_{P W F}=\mathbf{k}^{\dagger}[\widehat{M}]_{F P}^{-1} \mathbf{k} .
$$

One can observe that the span estimator from Eq. 17 has the same form as the Polarimetric Whitening Filter (PWF) introduced by Novak et al. in [11]. The only difference is the use of the normalized coherency estimate given by the Fixed Point estimator instead of the conventional Sample Covariance Matrix.

Finally, it is possible to derive an estimate of the conventional polarimetric coherency matrix according to Eq. 6:

$$
[\widehat{T}]_{F P}=\frac{\widehat{P}_{P W F}}{m}[\widehat{M}]_{F P} .
$$

4) Gaussian model in the SIRV context: The multivariate Gaussian distribution presented in Sect. II-A is obviously a member of the SIRV class. Let us assume $N$ i.i.d. realizations of the target vector k. The SCM from Eq. 2 is the ML 
estimator of $[T]$ in Gaussian clutter, but not in clutter described by the product model [41]. In the specific case of completely correlated texture ( $\tau=\tau_{i}, \forall i \in\{1, . . N\}$ ), Richmond proved that SCM is again the exact ML estimator of $[M]$ provided the M-normalization is respected [42]. In fact, the completely correlated $\tau$ case is equivalent to the Gaussian model for a given realization of data across all resolution cells [31]. Consequently, it is possible to define the normalized Sample Covariance Matrix as:

$$
[\widehat{M}]_{S C M}=m \frac{[\widehat{T}]_{S C M}}{\operatorname{Tr}\left\{[\widehat{T}]_{S C M}\right\}}
$$

In other words, in Gaussian clutter the local power $P$ is no more random in Eq. 9, but $m \cdot \tau=P$ with probability one [31]. Based on this consideration and according to Eq. 17, the Multilook Polarimetric Whitening Filter (MPWF) can be defined as:

$$
\widehat{P}_{M P W F}=\frac{1}{N} \sum_{i=1}^{N} \mathbf{k}_{\mathbf{i}}^{\dagger}\left[\widehat{M}_{i}\right]_{F P}^{-1} \mathbf{k}_{\mathbf{i}} .
$$

MPWF is the span ML estimator for Gaussian clutter with known power $P$ and it is unbiased [13], [14]. When compared to the span estimator from Eq. 5, the main advantage of MPWF is that it takes into account the correlation between the different polarization channels (speckle) in the whitening process.

\section{Spatial support}

In the estimation process a certain number of samples must be gathered for deriving the observation vector. In this purpose, the boxcar sliding neighborhood (BN) is usually employed. The main drawback of BN non-adaptive neighborhood is that the available number of samples is directly proportional with the loss of spatial resolution. In order to deal with this undesired effect, several strategies to obtain locally Adaptive Neighborhoods (AN) were proposed for POLSAR data processing. In [8], three local neighborhoods are analyzed and their performances are discussed with respect to different end-user applications (visual interpretation, classification...). Experiments on real data sets have shown that the Intensity-Driven-Adaptive-Neighborhood (IDAN) represents, on the whole, a good trade-off between preserving signal characteristics and gathering a significant number of samples for coherency and $H-\alpha-A$ parameter estimation [8], [43].

Recent studies have revealed that the original IDAN algorithm tends to introduce a bias with respect to the radiometry information [44]. The main reasons are the use of a symmetric confidence interval around the mean for the Gamma distributed intensity and the estimation of the initial seed by the median computed within a $3 \times 3$ neighborhood. In order to deal with these problems the SDAN algorithm (Span-Driven-Adaptive-Neighborhood) has been introduced in [45]. It allows to use heterogeneous scene models, such as SIRV, in the estimation step. Note that this approach is not optimal as the resulting AN is driven on the texture (span) information only. One may use other existing locally adaptive neighborhoods (e.g. directional neighborhoods [9]) but, up to now, the existing AN algorithms are also tributary to the span information.

SDAN successively truncates the texture PDF using two symmetric confidence intervals around the mean. The truncation thresholds are expressed with Gamma-distributed texture. However, different PDFs can be truncated according to the same thresholds (initially set using a Gamma prior). In this paper, the SDAN is employed to eliminate eventual outliers from the local neighborhood. The main advantage of this approach consist in selecting spatially connected pixels within a certain confidence interval. Its main inconvenient is the estimation bias which can be induced by truncating the significant part of the unknown texture PDF. 
Within the SIRV context, the SDAN algorithm operates under deterministic texture hypothesis: if $\tau$ is deterministic, the span statistics over "matrix stationary" areas is given by the Gamma PDF resulting from the complex Gaussian kernel. This is coherent with the general hypothesis adopted for POLSAR speckle filtering stating that the local "matrix stationarity" property is revealed by changes in the span image when texture is absent [9].

\section{Application to POLSAR parameter estimation}

One way to derive the normalized coherency matrix is the normalized Sample Covariance estimator, obtained by locally replacing the statistical mean by spatial average within the sliding neighborhood $\mathcal{W}$ :

$$
[\widehat{M}]_{S C M}(i, j)=\frac{m}{\operatorname{Tr}\left\{[\widehat{T}]_{S C M}\right\}}[\widehat{T}]_{S C M} \quad \text { with } \quad[\widehat{T}]_{S C M}=\frac{1}{\operatorname{card}\{\mathcal{W}(i, j)\}} \sum_{(p, q) \in \mathcal{W}(i, j)} \mathbf{k}(p, q) \mathbf{k}^{\dagger}(p, q),
$$

where $(i, j)$ represent the current range/azimuth position and $\operatorname{card}\{\mathcal{W}\}$ denotes the cardinal of $\mathcal{W}$. The main advantage of the $[\widehat{M}]_{S C M}$ estimator consists in deriving the polarimetric covariance matrix independently of the span for the Gaussian case. The normalized SCM estimator presents also one major disadvantage: it is not "SIRP stationary" and, as a consequence, this estimator is not consistent over textured areas. Finally, although the derivation of the normalized SCM estimator from the standard SCM estimator is straightforward, we could not find any specific paper to report its use for POLSAR data.

In this paper, we propose to extend the estimation of the normalized polarimetric coherency matrix by using a heterogeneous scene model over the sliding neighborhood. The Fixed Point estimator of the normalized covariance matrix for the SIRV model is applied using the procedure described in Sect. II-B. More precisely, the FP normalized coherency matrix is computed iteratively as:

$$
\left[\widehat{M}_{l}\right]_{F P}(i, j)=\frac{m}{\operatorname{card}\{\mathcal{W}(i, j)\}} \sum_{(p, q) \in \mathcal{W}(i, j)} \frac{\mathbf{k}(p, q) \mathbf{k}^{\dagger}(p, q)}{\mathbf{k}^{\dagger}(p, q)\left[\widehat{M}_{l-1}\right]_{F P}^{-1}(i, j) \mathbf{k}(p, q)} \quad \text { with } \quad\left[\widehat{M}_{0}\right]_{F P}=\left[I_{m}\right],
$$

where $l$ is the iteration index. Eq. 22 gives the covariance matrix estimate of the SIRV complex Gaussian kernel, without imposing any statistical constraint over the texture random variable $\tau$. The resulting matrix $[\widehat{M}]_{F P}$ is asymptotically Gaussian distributed. The proposed procedure (SDAN-FP) starts by computing the adaptive neighborhood using the SDAN algorithm presented in Sect. II-C at each range/azimuth position. The resulting adaptive neighborhood is supposed to respect the "matrix stationarity" condition. Finally, the FP estimator is applied to derive the normalized polarimetric coherency matrix estimate under compound Gaussian polarimetric clutter model (Eq. 22).

Another physical parameter to be estimated is the total power. For the SIRV model, the PWF span estimator is the ML estimator, hence it should be applied for textured areas. However, on Gaussian textureless areas, a stronger speckle reduction can be obtain using the MPWF estimator. In practical applications, the PWF and the MPWF estimators should be applied as follows: on "Gaussian stationary" regions the best span estimator is the MPWF, while on "SIRV homogeneous" areas only, the PWF should be applied. We propose to deal with this trade-off by applying the LLMMSE criterion for the span estimation [9]:

$$
\widehat{P}_{L L M M S E}=\widehat{P}_{M P W F}+\alpha_{L L M M S E}\left(\widehat{P}_{P W F}-\widehat{P}_{M P W F}\right) \quad \text { with } \quad \alpha_{L L M M S E}=\frac{\sigma_{P W F}^{2}\left(1+\sigma_{n}^{2}\right)}{\sigma_{M P W F}^{2}-\mu_{M P W F}^{2} \sigma_{n}^{2}},
$$

where $\mu_{M P W F}, \sigma_{P W F}, \sigma_{M P W F}$ are respectively the signal mean and standard deviations computed inside the local estimation neighborhood and where $\sigma_{n}$ is the noise standard deviation ("a priori" known). In Eq. 23, the two span estimators can be computed according to Eqs. 17 and 20. 
In the last stage, it also possible to unify these two descriptors by multiplying them according to the SIRV model from Eq. 6. An important remark is that by multiplying the two descriptors the separation between the total received power (span) and the polarimetric information (speckle normalized coherency) is lost. Finally, the resulting coherency matrix $[\widehat{T}]$ does not obey the Wishart PDF as it depends on the estimated span PDF.

[Figure 1 about here.]

In summary, this section introduces a novel estimation scheme (see Fig. 1) for deriving normalized polarimetric coherency matrices and resulting estimated span. The proposed algorithm couples span-driven multi-resolution techniques [45] with heterogeneous SIRV scene models [20] to deal with the polarimetric texture inside the estimation neighborhood. It is important to notice that the proposed Fixed Point estimator uses normalized coherency matrix inversion and thus it works only with Hermitian positive definite normalized coherency matrices. This constraint is still acceptable since, in practice, image coherency matrices are generally of full rank (3 for monostatic POLSAR data) [46]. However, in the specific case of a non-invertible matrix, which can correspond to a strongly polarized scattered signal, the SIRV model can be applied by using only the nonzero signal subspace.

\section{E. Distance measures for POLSAR segmentation}

Classification of ground cover with POLSAR data is an important application [17], [18], [19], [47], [48], [49]. Generally, one has to find a distance between the pixel covariance matrix $[C]$ and the class center $[C]_{\omega}$. Based on this distance, conventional clustering methods have already been introduced with POLSAR data: "naive” Bayesian ML classifier or K-means [18], fuzzy K-means or Expectation-Maximization [47].

When the POLSAR data are modelled by a stochastic process with a known PDF, it is possible to derive optimal ML distance measures (e.g. the Wishart distance for Gaussian processes). In [27], Yueh et al. derived an optimal ML distance measure for terrain cover classification using the normalized target vector in the lexicographical basis. The adopted normalization condition was the Euclidian norm and the distance measure was computed applying the Bayesian ML classifier with the PDF of the normalized polarimetric data. Note that in Yueh's approach, the covariance matrix is estimated using the sample covariance matrix (ML estimator only with Gaussian clutter). In consequence, the derived optimal distance is a Generalized Maximum Likelihood distance for Gaussian clutter only.

We propose the following general binary hypothesis test for a given class $\omega$ :

$$
\left\{\begin{array}{l}
H_{0}:[C]=[C]_{\omega} \\
H_{1}:[C] \neq[C]_{\omega}
\end{array}\right.
$$

According to the Neyman-Pearson Lemma, the LRT (Likelihood Ratio Test) provides the most powerful test [50]:

$$
\Lambda=\frac{p_{m}\left(\mathbf{k}_{1}, \ldots, \mathbf{k}_{N} / H_{1}\right)}{p_{m}\left(\mathbf{k}_{1}, \ldots, \mathbf{k}_{N} / H_{0}\right)} .
$$

For Gaussian clutter, maximizing the LRT from Eq. 25 and replacing the pixel coherency matrix $[T]$ with the ML estimate 
$[\widehat{T}]_{S C M}$ is equivalent to minimizing the the conventional Wishart distance:

$$
D_{\text {Wishart }}\left([\widehat{T}]_{S C M},[T]_{\omega}\right)=\ln \frac{\operatorname{det}\left\{[T]_{\omega}\right\}}{\operatorname{det}\left\{[\widehat{T}]_{S C M}\right\}}+\operatorname{Tr}\left\{[T]_{\omega}^{-1}[\widehat{T}]_{S C M}\right\} .
$$

This distance has been widely used for supervised and unsupervised POLSAR data clustering [18], [19], [47].

In the case of the SIRV model, one can rewrite the hypothesis test as:

$$
\left\{\begin{array}{lll}
H_{0}:[M]=[M]_{\omega} & \Leftrightarrow \quad \mathbf{k}=\sqrt{\tau} \mathbf{z}, \text { with } \mathbf{z} \sim \mathcal{N}(0,[M]) \\
H_{1}:[M] \neq[M]_{\omega} & \Leftrightarrow \quad \mathbf{k}=\sqrt{\tau} \mathbf{z}, \text { with } \mathbf{z} \sim \mathcal{N}\left(0,[M]_{\omega}\right)
\end{array}\right.
$$

where $\tau$ is the unknown deterministic texture.

For a given class $[M]_{\omega}$, the LRT with respect to the texture $\tau$ and the normalized coherency matrix $[M]$ is given by:

$$
\Lambda_{S I R V}=\frac{\prod_{n=1}^{N} \frac{1}{\pi^{m} \tau_{n}^{m} \operatorname{det}\left\{[M]_{\omega}\right\}} \exp \left\{-\frac{\mathbf{k}_{n}^{\dagger}[M]_{\omega}^{-1} \mathbf{k}_{n}}{\tau_{n}}\right\}}{\prod_{n=1}^{N} \frac{1}{\pi^{m} \tau_{n}^{m} \operatorname{det}\{[M]\}} \exp \left\{-\frac{\mathbf{k}_{n}^{\dagger}[M]^{-1} \mathbf{k}_{n}}{\tau_{n}}\right\}} .
$$

Notice the likelihood function in Eq. 28 does not use the stochastic texture description as the PDF $p(\tau)$ is supposed unknown in the SIRV model. As previously stated in Sect. II-B.3, the texture parameter $\tau$ can be considered either as a random variable with unknown PDF $p(\tau)$ or as an unknown deterministic parameter with PDF $p(\tau)=\delta\left(\tau-\tau_{n}\right)$ which characterizes yet a particular SIRV process. It can be shown that the ML estimation of the coherency matrix yields a good approximate ML estimate in the first case and the true ML estimate in the second case [31], [38] . The general PDF being unknown, it is therefore impossible to derive a texture independant closed-form expression for the Likelihood Ratio of the test given by Eq. 27. This procedure is here simplified, considering a particular SIRV process with a texture characterized by an unknown deterministic parameter. Consequently, each resolution cell is now associated with its own $p(\tau)=\delta\left(\tau-\tau_{n}\right)$ in Eq. 7, where $\tau_{n}$ are the unknown deterministic texture variables. This way the texture descriptor can be discarded for each pixel independently.

By taking the natural logarithm in Eq. 28, one obtains:

$$
\ln \left(\Lambda_{S I R V}\right)=-N \ln \frac{\operatorname{det}\left\{[M]_{\omega}\right\}}{\operatorname{det}\{[M]\}}-\sum_{n=1}^{N} \frac{\mathbf{k}_{n}^{\dagger}\left([M]_{\omega}^{-1}-[M]^{-1}\right) \mathbf{k}_{n}}{\tau_{n}} .
$$

Now, since the $\tau_{n}$ 's and $[M]$ are unknown, they are replaced by their ML estimates from Eq. 11 and Eq. 13. The resulting Generalized Likelihood Ratio Test $\Lambda_{S I R V}^{\prime}$ is given by:

$$
\ln \left(\Lambda_{S I R V}^{\prime}\right)=-N \ln \frac{\operatorname{det}\left\{[M]_{\omega}\right\}}{\operatorname{det}\left\{[\widehat{M}]_{F P}\right\}}-m \sum_{n=1}^{N} \frac{\mathbf{k}_{n}^{\dagger}[M]_{\omega}^{-1} \mathbf{k}_{n}}{\mathbf{k}_{n}^{\dagger}[\widehat{M}]_{F P}^{-1} \mathbf{k}_{n}}+N m .
$$

Maximizing the generalized LRT over all classes is equivalent to minimizing the following SIRV distance:

$$
D_{S I R V}\left([\widehat{M}]_{F P},[M]_{\omega}\right)=\ln \frac{\operatorname{det}\left\{[M]_{\omega}\right\}}{\operatorname{det}\left\{[\widehat{M}]_{F P}\right\}}+\frac{m}{N} \sum_{n=1}^{N} \frac{\mathbf{k}_{n}^{\dagger}[M]_{\omega}^{-1} \mathbf{k}_{n}}{\mathbf{k}_{n}^{\dagger}[\widehat{M}]_{F P}^{-1} \mathbf{k}_{n}} .
$$

Notice that computing the distance from Eq. 31 needs the original scattering vectors $\mathbf{k}_{n}$.

In this paper, the distance measure from Eq. 31 is used as a dissimilarity measure in the conventional K-means clustering for POLSAR data. The full description of the K-means algorithm can be found in [18]. 
In summary, this section introduces a new distance measure between normalized coherency matrices. The resulting approximate generalized ML distance is optimal, in the generalized LRT sense, for POLSAR data characterized by the SIRV model.

An interesting remark concerning the SIRV distance can be observed in Eq. 31. On one hand, when the texture (span information) is high, the second term of the SIRV distance $D_{S I R V}$ becomes small and the distance measure is dominated by the determinants ratio. This usually corresponds to strongly polarized targets with a dominant scattering mechanism (eg. dihedral, trihedral ...). On the other hand, with smaller span values, the distance is dominated by the second term which takes into account the $N$ observed samples. This second case often corresponds to distributed targets.

\section{RESULTS AND DISCUSSION}

This section has two main objectives. The first one consists in evaluating the performance of the normalized coherency estimation techniques presented in Sect. II. The second objective is to show the improvement in the conventional POLSAR processing chain brought by introducing the normalized coherence matrix related to the SIRV model.

Three different estimation techniques are analyzed: the normalized Sample Covariance Matrix coupled with the $7 \times 7$ Boxcar Neighborhood (BN-SCM) and the Fixed Point estimator coupled either with the $7 \times 7$ Boxcar Neighborhood (BN-FP) or with the SDAN adaptive neighborhood (SDAN-FP). In all three cases, the corresponding span image is estimated using the LLMMSE estimator from Eq. 23. The parameters used for the SDAN algorithm are $L_{e q}=3$ and $N_{\max }=50$. Note that $N_{\max }$ is limiting the number of samples for the SDAN region growing only. The testing of SDAN background pixels is not limited to a fixed number of samples to approximate the final i.i.d. condition (see Appendix A).

\section{A. Simulated POLSAR data}

As, for real data, it is impossible to find reference regions with known coherency matrix, the effectiveness of the estimation schemes is demonstrated using simulated POLSAR data [30].

1) Gaussian case: The first POLSAR data set consists of four adjacent Gaussian regions as presented in Fig. 2. Each of the four quadrants is associated with a known deterministic texture value [Fig. 2-(a)] and a known theoretical covariance matrix. Using these parameters each component of the polarimetric target vector is simulated accordingly. Fig. 2-(b) illustrates the initial span image computed using Eq. 3 and Fig. 2-(c) shows the resulting amplitude color composition of the three target vector components.

[Figure 2 about here.]

The LLMMSE span $P$ and the normalized coherency matrix $[M]$ are estimated using the three different estimation schemes. Note that in the Gaussian case, the optimal ML estimation technique is the BN-SCM from Fig. 3-(a) and Fig. 3-(d). Inside each quadrant, the stochastic process characterizing the data is "Gaussian stationary", hence it is also "SIRV homogeneous". The BN-FP estimation yields quite similar results, from the visual point of view, as illustrated in Fig. 3-(b) and Fig. 3-(e). However, the use of the BN is associated with the well-known edge-blurring effect as the "matrix stationarity" condition is not respected 
over the transitions between the quadrants. The SDAN-FP estimation reduces this undesired effect as presented in Fig. 3-(c) and Fig. 3-(f). As a general remark, the blurring is more present within the normalized coherency diagonal elements than within the span images due to the use of the adaptive LLMMSE span estimator.

[Figure 3 about here.]

In order to objectively asses the estimation performances, the mean and the variance for each element of the normalized coherency are computed over the SE quadrant. A global error measure $\epsilon$ for the normalized coherency matrix is also introduced as:

$$
\epsilon=\frac{1}{N} \sum_{i=1}^{N} \frac{\left\|\left[\widehat{M}_{i}\right]-\left[M_{r e f}\right]\right\|_{F}}{\left\|\left[M_{r e f}\right]\right\|_{F}} .
$$

where $\left[M_{r e f}\right]$ is the reference normalized coherency matrix used for data simulation. As observed in Tab. I, the best results are obtained using the BN-SCM estimator. Being the ML estimator for "Gaussian stationary" regions, the SCM is used as benchmark for the SDAN neighborhood and the FP estimator. The mean value is well preserved for both BN-FP and SDAN-FP estimates, while the variance of the BN-FP is smaller than the measured variance of the SDAN-FP. The latter observation is explained by the fact that the boxcar neighborhood is optimal on such "SIRV homogeneous" regions. One can also note that, despite the mean of each element of the normalized coherency being quite similar, a better error measure is provided by the $\epsilon$ parameter. Using the Frobenius norm, which is a norm associated to the inner product on the ring of all complex matrices, the corresponding error $\epsilon$ shows that the smallest error is obtained for the optimal BN-SCM estimator. When introducing the FP estimator, $\epsilon$ increases and it increases even more by using the SDAN adaptive spatial support. This behavior corresponds to the expected theoretical observations. However, it is important to notice that for both BN-FP and SDAN-FP the error is not increased by more than $7 \%$ with respect to the ML estimator. This is acceptable for the POLSAR applications where the clutter is characterized by a "Gaussian stationary" stochastic process.

[Table 1 about here.]

A similar objective performance assessment is carried out for the estimation of the span image. Tab. II shows the span mean ratio and the speckle coefficient of variation computed for the same "Gaussian stationary" region. As for the normalized coherency, the bias in the estimated radiometry is less than $7 \%$ for all three estimation techniques. An interesting remark consists in the fact that, when using the Fixed Point estimator, the bias from Tab. II is also linked with the average computed over the corresponding homogeneous region. Even if the mean ratio is a standard parameter for evaluating the speckle filter radiometric bias, this parameter is not so well adapted for the Fixed Point estimation. Although being asymptotically Gaussian distributed, the FP estimator is outperformed by the SCM (ML estimator for Gaussian clutter) with a fixed number of samples. Consequently, the average over homogeneous area should be coupled with the estimation of the FP normalized coherency over the same homogeneous population for optimal performances. This effect can be noticed with the SDAN-FP span, where the local SDAN neighborhood can gather more than 49 samples over "Gaussian stationary" areas. The resulting SDAN-FP span estimator exhibits a radiometric bias less than $1 \%$ (same as for BN-SCM).

[Table 2 about here.] 
In summary, subjective and objective performance assessment carried out for Gaussian POLSAR clutter shows that, despite being suboptimal, the proposed Fixed Point estimator and the adaptive SDAN neighborhoods give good overall performances. The corresponding error measure is less than $7 \%$ for all estimation schemes.

2) SIRV case: The second simulated POLSAR data set proposes the same four quadrants, but with Gamma distributed texture [Fig. 4-(a)]: each quadrant is $\mathcal{K}$-distributed. The texture coefficient of variation used for simulation equals 3 , which corresponds to a highly non-Gaussian clutter (urban areas). Fig. 4-(b) presents the initial span image. Fig. 4-(c) shows the corresponding amplitude color composition of the three target vector components.

[Figure 4 about here.]

The overall data set is not "SIRV homogeneous" as the "matrix stationarity" condition is not respected on the boundaries, however each quadrant is "SIRP stationary". In the following, we shall use only the "SIRV homogeneity" assumption over each quadrant, namely the texture PDF is supposed unknown. Fig. 5 illustrates the LLMMSE span $P$ and the normalized coherency matrix $[M]$ estimated using the three different estimation schemes.

[Figure 5 about here.]

As the data set is not Gaussian, the PWF span estimator is dominant in the LLMMSE criterion and the corresponding speckle reduction is performed using only three samples. Hence, concerning the LLMMSE span estimation, BN-SCM, BN-FP and SDAN-FP [Fig. 5-(a),(b),(c)] look similar from the visual point of view.

The effectiveness of the Fixed Point estimator in compound Gaussian clutter can be observed in Fig. 5-(e),(f). While the BN-SCM normalized coherency [Fig. 5-(d)] presents a "patchy" appearance, the BN-FP estimation [Fig. 5-(e)] provides better visual homogeneity within each quadrant. The adaptive SDAN spatial support [Fig. 5-(f)] assures better edge preservation for the transitions between quadrants. One important issue is that the diagonal elements of the BN-FP normalized coherency for the SIRV case [Fig. 5-(e)] have the same visual aspect as for the previous Gaussian POLSAR data set [Fig. 3-(e)]. This shows that the FP estimate of the covariance matrix does not depend on the texture PDF.

Using the same reference region as for Gaussian case, Tab. III presents the mean and the variance for each element of the normalized coherency, and also the overall error measure $\epsilon$ computed for the three estimation schemes. BN-FP and SDAN-FP outperform BN-SCM in retrieving the reference value and also in terms of variance reduction. Since the "matrix stationarity" is always assured within the reference region, BN-FP outperforms the SDAN-FP also. Finally, another interesting result consists in the fact that Tab. III indicates the same BN-FP value for the $\epsilon$ error parameter as in the Gaussian case (Tab. I). This objective issue confirms the visual comparison mentioned in the previous paragraph.

[Table 3 about here.]

[Table 4 about here.] 
Objective performance assessment has been carried out for the LLMMSE span estimation also. Tab. IV presents the Kolmogorov-Smirnov (KS) test with respect to the reference span used for simulation. The resulting KS values, computed over the entire span image, indicate that BN-FP outperforms BN-SCM. The best results are reported when using the SDAN-FP estimator. Note that the KS distance is rather small $\epsilon \in(0.07,0.12)$ in all three cases.

\section{B. Airborne POLSAR data}

To illustrate the improvements in the standard POLSAR processing chain, results obtained with high and very high resolution airborne data are reported. Both data sets were acquired by the airborne ONERA RAMSES system [51].

1) High resolution POLSAR data: The first POLSAR data set was acquired in Brétigny, France. The mean incidence angle is $30^{\circ}$. It represents a fully polarimetric (monostatic mode) X-band acquisition with a spatial resolution of approximately $1.5 \mathrm{~m}$ in range and azimuth.

[Figure 6 about here.]

Fig. 6-(a) presents the color composition of target vector amplitudes. The target area is composed of three buildings, a parking lot and the surrounding agricultural areas. For further illustration, a non-Gaussian urban (building) region has been selected, namely the span image superposed over the airborne photo from Fig. 6-(b).

The LLMMSE span and the normalized coherency matrix are estimated using the three different estimation schemes [Fig. 7]. The BN-FP span illustrated in Fig. 7-(b) exhibits better whitening in the estimation process than the BN-SCM span from Fig. 7(a). This can be observed on the isolated brilliant pointwise structures surrounding the building. However, both BN-SCM and BN-FP are tributary to the "ring effect" (two large dips on a spatial profile near the boundaries of a pointwise target) induced by coupling the BN spatial support with the LLMMSE estimator [52]. This effect is reduced in the SDAN-FP span image as it can be observed over the metallic structures present on the roof of the building from Fig. 7-(c).

\section{[Figure 7 about here.]}

Visual assessment is carried out also with normalized coherency $[M]$ estimates. Color compositions, constructed from either the diagonal elements of $[M]$ or the corresponding $H-\alpha-A$ parameters [17], are computed for the three estimation techniques [Fig. 7-(d),(e),(f) and Fig. 7-(g),(h),(i)]. Both parameters exhibit the same behavior:

- BN-SCM: "patchy" appearance mainly due to the texture;

- BN-FP: blurring as the "matrix stationarity" condition is not respected;

- SDAN-FP: higher spatial feature preservation but more variance.

As the target area is highly heterogeneous, the SDAN-FP estimation is a good trade-off between robust estimation and spatial resolution preservation.

Finally, it is possible to derive the SDAN-FP polarimetric coherency matrix as the product between the span image [Fig. 7-(c)] and the corresponding normalized coherency [Fig. 7-(f)]. In Fig. 8, the SDAN-FP coherency is compared with the conventional 
coherency matrices obtained by the sample covariance matrix estimator coupled with two spatial supports: the boxcar neighborhood (BN) and the Intensity-Driven-Adaptive-Neighborhood (IDAN) [8]. Subjective visual assessment can be expressed in terms of the hue-saturation-lightness (HSL) color space [53] by associating the lightness to the span and the saturation to the polarimetric diversity. The SDAN-FP coherency from Fig. 8-(c) exhibits better performances in terms of lightness and saturation, meaning that both the span and the normalized coherency are better estimated. The corresponding $H-\alpha$ classification maps [17] are illustrated in Fig. 8-(d),(e),(f). For $H-\alpha$ classification also, the SDAN-FP coherency provides better performances as it achieves stronger noise reduction than the IDAN filter.

[Figure 8 about here.]

One key issue to be discussed is weather the normalized coherency matrix and the span should be aggregated in the final estimation step or not (the question mark from Fig. 1). Most of the existing processing chains use the conventional coherency matrix for representing POLSAR data for unsupervised land cover classification [18], [19], [47], [43] and for target detection applications [33], [12]. Due to the SIRV model identification problem discussed in Sect. II-B, the complete description of the POLSAR data set is achieved by estimating the span and the normalized coherency independently. The latter describes the polarimetric diversity, while the span indicates the total received power. Moreover, the Fixed Point estimation of the normalized coherence does not depend on the span information. Given these facts, we propose to investigate this problem in the framework of unsupervised POLSAR classification. The classification scheme discussed in the following is the standard Wishart $H-\alpha$ segmentation [18]. For segmenting the normalized coherency, we have modified the Wishart $H-\alpha$ algorithm by replacing the Wishart distance with the SIRV ML distance discussed in Sect. II-E. For comparison, we have also used the scalar Gamma K-means classification with $H-\alpha$ initialization. The corresponding ML distance measure is obtained using the GLRT with the Gamma PDF.

[Figure 9 about here.]

Fig. 9 illustrates the POLSAR unsupervised classification results using three descriptors estimated by SDAN-FP: span [Fig. 9(a)], coherency [Fig. 9-(b)] and normalized coherency [Fig. 9-(c)]. The selected scene is composed of both Gaussian (agricultural fields) and non-Gaussian (urban) areas. This case is encountered in many practical POLSAR classification applications. Fig. 9-(e) presents the 8-class segmentation map obtained using the SDAN-FP coherency matrix. When compared to the scalar unsupervised classification map [Fig. 9-(d)] obtained using span only, one can observe the high degree of similarity between them. This leads to the following conclusion: the Wishart $H-\alpha$ classification is mainly influenced by the information contained in the span image. Regarding the polarimetric information, Fig. 9-(f) presents the classification map computed using the normalized coherency matrix and the associated SIRV distance. The visual assessment of Fig. 9-(e) and Fig. 9-(f) reveals that a significant part of the polarimetric information is lost when using the standard coherency matrix: the building class separation is lost, as well as the natural canonical targets (trihedral, dihedral...) present over different "field" classes. One important remark concerning the Wishart $H-\alpha$ classification is that a large number of samples are usually assigned to the class feature vector when iterating the K-means clustering algorithm. Due to this, locally Gaussian areas (agricultural fields) may become heterogeneous regions as neither the "matrix stationarity" nor the "texture homogeneity" conditions are respected. 
The same behavior can be also observed in Fig. 9-(g),(h),(i) with the classification maps obtained after basic scattering mechanism identification [54]. The use of polarimetric indicators, derived from the eigenvector-eigenvalue decomposition of the normalized coherency matrix, allows the interpretation of each cluster scattering mechanism from Fig. 9-(d),(e),(f). In all three cases, the POLSAR parameters were computed using the SDAN-FP normalized coherency from Fig. 9-(f). The observed scene is then classified into three canonical scattering types: even bounce (blue or cyan), odd bounce (red or dark red) and volume scattering (green) [55].

2) Very high resolution POLSAR data: The second POLSAR data (Fig. 10) set was acquired in Toulouse, France with a mean incidence angle of $50^{\circ}$. It represents a fully polarimetric (monostatic mode) X-band acquisition with a spatial resolution of approximately $50 \mathrm{~cm}$ in range and azimuth.

[Figure 10 about here.]

Fig. 11 presents the visual assessment of the proposed estimation scheme applied to very high resolution POLSAR data acquired in urban environment. The obtained results are visually compared to those obtained by the refined Lee filter operating under Gaussian clutter hypothesis [9]. With a $50 \mathrm{~cm}$ spatial resolution, the SDAN-FP normalized coherency from Fig. 11-(c) reveals higher variability in polarimetric signatures than with a $1.5 \mathrm{~m}$ spatial resolution [Fig. 7-(f)]. Fig. 11-(b) presents the color composition of the diagonal elements of the SDAN-FP coherency matrix after multiplication by the corresponding LLMMSE span. When compared to the polarimetric coherency derived by the refined Lee filter [Fig. 11-(a)], the SDAN-FP coherency better preserves the polarimetric and radiometric signatures over thin spatial features (brilliant points and edges). This can be also observed in the 8-class segmentation maps obtained by Wishart $H-\alpha$ clustering [Fig. 11-(d),(e)]. It is important to stress that, for very high resolution urban POLSAR data, the polarimetric coherency matrix is not Wishart distributed. Hence, the unsupervised $H-\alpha$ classification based on the SIRV distance measure can be properly applied using the SDAN-FP normalized coherency. The result is illustrated in Fig. 11-(f). Finally, the three classification maps are interpreted according to the basic scattering mechanism identification procedure [54]. Subjective visual assessment indicates that quite realistic results are obtained using the SDAN-FP normalized coherency descriptor [Fig. 11-(i)]: buildings and cars are mainly retrieved in the red "odd bounce" class, while "even bounce" scattering mechanism (cyan class) appears on the flat regions (roads).

[Figure 11 about here.]

In conclusion, the joint analysis of the span and the normalized coherency presents several advantages with respect to the coherency matrix descriptor: separation between the total received power and the polarimetric information, estimation of the normalized coherency matrix independently of the span and the existence of the SIRV distance measure for unsupervised ML classification of normalized coherencies. However, the span-normalized coherency description of POLSAR images raises new problems which still remain under investigation. The first issue concerns the use of span for testing the "matrix stationarity" condition for the normalized coherency estimation. This test is currently used for POLSAR data speckle filtering and it is founded on the basic principle that changes within the polarimetric signature are revealed by changes in the total received power. Consequently, one may envisage other estimation schemes dedicated to the SIRV model with stochastic texture by considering external estimators of "matrix stationarity". The second important remark concerns the Wishart unsupervised 
classification scheme. Although all statistical requirements employed for unsupervised classification are met, the polarimetric information is quite difficult to extract using the K-means clustering. As it can be noticed in Fig. 9-(c), the polarimetric signatures are strongly mixed and the class boundaries are smoothed within high resolution POLSAR images (even for highly heterogeneous target areas). Therefore, other clustering strategies should be better suited to capture the spatial distribution of different polarimetric signatures. One starting point could be the POLSAR segmentation by likelihood approximation [56], spectral clustering ensemble [57], or the Support Vector Machines kernel-based non-linear classification [58].

Finally, one can observe that span information does also, in some cases, contribute to classification quality (e.g. discrimination of roads in Fig. 9 and buildings in Fig. 11), although the polarimetric signature clustering suffers. Based on the SIRV model, the separation span/polarimetric signature is achieved. Future work is needed to objectively asses the classification potential of these two descriptors separately.

\section{General Remarks}

One critical point of the SIRV model is linked to the scalar texture (span) descriptor $\tau$. The validity of the product model for POLSAR data has been investigated in many papers over the last decades [28], [11], [12], [7], [13].

Yueh et al. derived the generalized likelihood of the normalized polarimetric target vector in Gaussian clutter [27]. This approach has been extended to the $\mathcal{K}$-distributed clutter in [6], [28]. Note that this extension is not optimal since the covariance matrix parameter is replaced by the Sample Covariance Matrix. Or the SCM depends on the texture PDF $p(\tau)$ and it is not the ML estimator of the covariance matrix in $\mathcal{K}$-distributed clutter. The exact ML normalized covariance estimator can be derived using the Yao's representation theorem for SIRVs and its exact expression is given in Eq. 16.

The product model have also been also used by Novak et al. for deriving the Polarimetric Whitening Filter [11], [12]. Based on this result, Lopes et al. derived the Multilook Polarimetric Whitening Filter as well as the adaptive LLMMSE filter for Gaussian and $\mathcal{K}$-distributed clutter [13]. The SIRV representation theorem allows the derivation of the PWF as a ML estimator of the deterministic texture. Once the texture parameter is obtained for every resolution cell further statistical processing can be applied over a population of texture parameters (e.g. the proposed LLMMSE span filter).

For Gaussian clutter, Lee at al. introduced optimal polarimetric covariance matrix classification schemes based on the Wishart distance [18], [19], [47]. The proposed methods can be extended to the SIRV model by using the SIRV distance presented in Sect. II-E. Moreover, the asymptotical distribution of the Fixed Point estimator from Eq. 13 has been derived in [39]. The FP estimator computed with $N$ samples (secondary data) converges in distribution to the normalized Sample Covariance Matrix computed with $N \frac{m}{m+1}$ secondary data. Since the normalized SCM is the SCM up to a scale factor, we may conclude that, in problems invariant with respect to a scale factor on the covariance matrix, the FP estimate is asymptotically equivalent to the SCM computed with $N \frac{m}{m+1}$ secondary data.

We can conclude that the Yao's representation theorem allows optimal multivariate signal processing of POLSAR data in a general framework. The SIRV model provides the methodology for retrieving the conventional cases (multivariate Gaussian and multivariate $\mathcal{K}$-distribution). This methodology can be also generalized to other heterogeneous clutter models defined by explicit texture PDFs (inverse Gamma, Fisher ...). 
More recent studies have revealed the presence of different scattering characteristics between the cross-polar and co-polar terms of the Sinclair matrix [59], [16]. In consequence, the POLSAR clutter could be modelled by different texture random variables for each polarization channel. Such a stochastic model already exists in the literature and it is known as the Generalized SIRV model [60]. Unfortunately, the covariance matrix Generalized SIRV estimator of the Gaussian kernel could not be found, without taking into account any "a priori" information about the texture multivariate PDF. Future work should investigate the coupling between SIRVs and multiple single-channel spatial texture descriptors, such as the nonstationary anisotropic Gaussiankernel model [61].

Despite being quite general, the SIRV clutter model supposes the "matrix stationarity" condition to be verified over the observation vector. We proposed the use of an adaptive spatial support based on the scalar span information. The resulting SpanDriven-Adaptive-Neighborhood operates under deterministic texture hypothesis and it states that the local "matrix stationarity" property is revealed by changes in the span image.

One limitation of the proposed estimation scheme concerns the determination of the "SIRV homogeneous" neighborhood surrounding a pixel. The strategy adopted for this paper consist in testing the "matrix stationarity" condition using the span, under deterministic texture assumption. Despite not being optimal in the context of the SIRV model, the proposed approach does not require additional "a priori" information regarding the local clutter statistics.

Finally, the SDAN-FP algorithm is more computation intensive than other existing POLSAR speckle filtering algorithms developed for Gaussian clutter [9], [8], [10] and it handles SIngle Look Complex data only. Further work should address the extension of the proposed approach to adaptive nonlinear filtering of multilook POLSAR data.

\section{CONCLUSiOnS AND PERSPECTIVES}

This paper presented a new estimation scheme for deriving normalized coherency matrices and the resulting estimated span with high resolution POLSAR images. The proposed approach couples nonlinear ML estimators with span driven adaptive neighborhoods for taking the local scene heterogeneity into account.

The heterogeneous clutter in POLSAR data was described by the SIRV model. Two estimators were introduced for describing the POLSAR data set: the Fixed Point estimator of normalized coherency matrix and the corresponding LLMMSE span. The Fixed Point estimation is independent on the span PDF and represents an approximate ML estimator for a large class of stochastic processes obeying the SIRV model. Moreover, the derived normalized coherency is asymptotically Gaussian distributed.

For SIRV clutter, a new ML distance measure was introduced for unsupervised POLSAR classification. This distance was used in conventional K-means clustering initialized by the $H-\alpha$ polarimetric decomposition. Other extensions of the existing unsupervised or supervised POLSAR clustering methods (e.g. Bayes ML or fuzzy K-means) can be derived by replacing the conventional Wishart distance with the proposed SIRV distance.

The effectiveness of the proposed estimation scheme was illustrated by high and very high resolution ONERA RAMSES X-band POLSAR data. The reliability of the obtained results was demonstrated by quantitative performance assessments using simulated POLSAR data. 
This work has many interesting perspectives. We believe that this paper contributes toward the description and the analysis of heterogeneous clutter over scenes exhibiting complex polarimetric signatures. The proposed approach presents a high degree of generality as no explicit stochastic texture model is needed. Finally, the proposed estimation scheme can be extended to other multidimensional SAR techniques using the covariance matrix descriptor, such as: multi-baseline interferometry, polarimetric interferometry or multi-frequency polarimetry. Future work should address the quantitative performance analysis of classification and target detection algorithms based on these estimators.

\section{ACKNOWLEDGMENT}

This work was supported by Centre National d'Etudes Spatiales (CNES), the French Space Agency. The authors would like to thank the anonymous reviewers for helpful comments and suggestions. 


\section{REFERENCES}

[1] W. M. Boerner, "Basic concepts of radar polarimetry and its applications to target discrimination, classification, imaging, and identification," Communications Laboratory, University of Illinois, Chicago, USA, Tech. Rep. EMID-CL-82-05-18-0, 1982.

[2] J. W. Goodman, "Some fundamental properties of speckle," J. Opt. Soc. Amer., vol. 53, no. 11, pp. 1145-1149, 1976.

[3] C. Lopez-Martinez and X. Fabregas, "Polarimetric SAR speckle noise model," IEEE Transactions on Geoscience and Remote Sensing, vol. 44, no. 10, pp. 2232-2242, 2003.

[4] M. S. Greco and F. Gini, "Statistical analysis of high-resolution SAR ground clutter data," IEEE Transactions on Geoscience and Remote Sensing, vol. 45, no. 3, pp. 566-575, 2007.

[5] F. T. Ulaby, F. Kouyate, B. Brisco, and T. H. L. Williams, “Textural information in SAR images," IEEE Transactions on Geoscience and Remote Sensing, vol. GE-24, no. 2, pp. 235-245, 1986.

[6] S. H. Yueh, J. A. Kong, J. K. Jao, R. T. Shin, and L. M. Novak, "K-distribution and polarimetric terrain radar clutter," Journal of Electromagnetic Waves and Applications, vol. 3, no. 8, pp. 747-768, 1989.

[7] J. S. Lee, D. L. Schuler, R. H. Lang, and K. J. Ranson, "K-distribution for multi-look processed polarimetric SAR imagery," in Proceedings of the IEEE International Geoscience and Remote Sensing Symposium, Pasadena, SUA, vol. 4, 1994, pp. $2179-2182$.

[8] G. Vasile, E. Trouvé, J. S. Lee, and V. Buzuloiu, "Intensity-Driven-Adaptive-Neighborhood technique for polarimetric and interferometric SAR parameters estimation," IEEE Transactions on Geoscience and Remote Sensing, vol. 44, no. 5, pp. 1609-1621, 2006.

[9] J. S. Lee, M. R. Grunes, and G. DeGrandi, "Polarimetric SAR speckle filtering and its impact on terrain classification," IEEE Transactions on Geoscience and Remote Sensing, vol. 37, no. 5, pp. 2363-2373, 1999.

[10] J. S. Lee, J. H. Wen, T. L. Ainsworth, K. S. Chen, and A. J. Chen, "Improved sigma filter for speckle filtering of SAR imagery," IEEE Transactions on Geoscience and Remote Sensing, vol. 47, no. 1, pp. 202-213, 2009.

[11] L. M. Novak and M. C. Burl, "Optimal speckle reduction in polarimetric SAR imagery," IEEE Transactions on Aerospace and Electronic Systems, vol. 26, no. 2, pp. 293-305, 1990

[12] L. M. Novak, M. C. Burl, and W. W. Irving, "Optimal polarimetric processing for enhanced target detection," IEEE Transactions on Aerospace and Electronic Systems, vol. 29, no. 1, pp. 234-244, 1993.

[13] A. Lopes and F. Sery, "Optimal speckle reduction for the product model in multilook polarimetric SAR imagery and the Wishart distribution," IEEE Transactions on Geoscience and Remote Sensing, vol. 35, no. 3, pp. 632-647, 1997.

[14] G. Liu, S. Huang, A. Torre, and F. Rubertone, "The Multilook Polarimetric Whitening Filter (MPWF) for intensity speckle reduction in polarimetric SAR images," IEEE Transactions on Geoscience and Remote Sensing, vol. 36, no. 3, pp. 1016-1020, 1998.

[15] G. DeGrandi, J. S. Lee, D. Schuler, and E. Nezry, "Texture and speckle statistics in polarimetric SAR synthesized images," IEEE Transactions on Geoscience and Remote Sensing, vol. 41, no. 9, pp. 2070-2088, 2003.

[16] G. DeGrandi, J. S. Lee, and D. Schuler, "Target detection and texture segmentation in polarimetric SAR images using a wavelet frame: theoretical aspects," IEEE Transactions on Geoscience and Remote Sensing, vol. 45, no. 11, pp. 3437-3453, 2007.

[17] S. R. Cloude and E. Pottier, "An entropy based classification scheme for land applications of polarimetric SAR," IEEE Transactions on Geoscience and Remote Sensing, vol. 35, no. 1, pp. 68-78, 1997.

[18] J. S. Lee, M. R. Grunes, T. L. Ainsworth, D. Li-Jen, D. L. Schuler, and S. R. Cloude, "Unsupervised classification using polarimetric decomposition and the complex Wishart classifier," IEEE Transactions on Geoscience and Remote Sensing, vol. 37, no. 5, pp. 2249-2258, 1999.

[19] J. S. Lee, M. R. Grunes, E. Pottier, and L. Ferro-Famil, "Unsupervised terrain classification preserving polarimetric scattering characteristics," IEEE Transactions on Geoscience and Remote Sensing, vol. 42, no. 4, pp. 722-731, 2004.

[20] F. Pascal, Y. Chitour, J. P. Ovarlez, P. Forster, and P. Larzabal, "Covariance structure maximum-likelihood estimates in compound Gaussian noise: existence and algorithm analysis," IEEE Transactions on Signal Processing, vol. 56, no. 1, pp. 34-48, 2008.

[21] K. Yao, "A representation theorem and its applications to spherically-invariant random processes," IEEE Transactions on Information Theory, vol. 19, no. 5, pp. 600-608, 1973.

[22] J. S. Lee, "Speckle analysis and smoothing of synthetic aperture radar images," Comp. Graph. Image Process., vol. 17, no. 1, pp. $24-32$, 1981.

[23] R. Touzi, "A review of speckle filtering in the context of estimation theory," IEEE Transactions on Geoscience and Remote Sensing, vol. 40, no. 11, pp. 2392-2404, 2002.

[24] R. Fjortoft and A. Lopes, "Estimation of the mean radar reflectivity from a finite number of correlated samples," IEEE Transactions on Geoscience and Remote Sensing, vol. 39, no. 1, pp. 196-199, 2001.

[25] J. S. Lee, M. R. Grunes, and S. A. Mango, "Speckle reduction in multipolarization, multifrequency SAR imagery," IEEE Transactions on Geoscience and Remote Sensing, vol. 29, no. 4, pp. 535-544, 1991.

[26] A. DeMaio, G. Alfano, and E. Conte, "Polarization diversity detection in compound-Gaussian clutter," IEEE Transactions on Aerospace and Electronic Systems, vol. 40, no. 1, pp. 114-131, 2004.

[27] H. A. Yueh, A. A. Swartz, J. A. Kong, R. T. Shin, and L. M. Novak, "Bayes classification of terrain cover using normalized polarimetric data," Journal of Geophysical Research, vol. 93, no. B12, pp. 15 261-15 267, 1988.

[28] H. A. Yueh, J. A. Kong, J. K. Jao, R. T. Shin, H. A. Zebker, T. LeToan, and L. M. Novak, Polarimetric remote sensing, chapter 4, J. A. Kong, Ed. PIER 3, Elsevier Science Publishing Co., 1990.

[29] R. Touzi and A. Lopes, "The principle of speckle filtering in polarimetric SAR imagery," IEEE Transactions on Geoscience and Remote Sensing, vol. 32, no. 5, pp. 1110-1114, 1994

[30] M. Rangaswamy, D. Weiner, and A. Ozturk, "Computer generation of correlated non-Gaussian radar clutter," IEEE Transactions on Aerospace and Electronic Systems, vol. 31, no. 1, pp. 106-116, 1995.

[31] F. Gini and M. V. Greco, "Covariance matrix estimation for CFAR detection in correlated heavy tailed clutter," Signal Processing, vol. 82, no. 12, pp. $1847-1859,2002$.

[32] N. R. Goodman, "Statistical analysis based on a certain multivariate complex gaussian distribution (an introduction)," Annals of Mathematical Statistics, vol. 34 , no. 1 , pp. 152-177, 1963.

[33] L. M. Novak, M. B. Sechtin, and M. J. Cardullo, "Studies of target detection algorithms that use polarimetric radar data," IEEE Transactions on Aerospace and Electronic Systems, vol. 25, no. 2, pp. 150-165, 1989.

[34] J. Goldman, "Detection in the presence of spherically symmetric random vectors," IEEE Transactions on Information Theory, vol. IT-22, no. 1, pp. 52-59, 1976.

[35] E. Conte, M. Lops, and G. Ricci, "Asymptotically optimum radar detection in compound-Gaussian clutter," IEEE Transactions on Aerospace and Electronic Systems, vol. 31, no. 2, pp. 617-625, 1995.

[36] F. Gini, "Sub-optimum coherent radar detection in a mixture of K-distributed and Gaussian clutter," IEE Proceedings Radar, Sonar and Navigation, vol. 144, no. 1, pp. 39-48, 1997.

[37] M. Rangaswamy, J. H. Michels, and D. D. Weiner, "Multichannel detection for correlated non-Gaussian random processes based on innovations," IEEE Transactions on Signal Processing, vol. 43, no. 8, pp. 1915-1922, 2005.

[38] E. Conte, A. DeMaio, and G. Ricci, "Recursive estimation of the covariance matrix of a compound-Gaussian process and its application to adaptive CFAR detection," IEEE Transactions on Image Processing, vol. 50, no. 8, pp. 1908-1915, 2002. 
[39] F. Pascal, P. Forster, J. P. Ovarlez, and P. Larzabal, "Performance analysis of covariance matrix estimates in impulsive noise," IEEE Transactions on Signal Processing, vol. 56, no. 6, pp. 2206-2216, 2008.

[40] Y. Chitour and F. Pascal, "Exact maximum likelihood estimates for SIRV covariance matrix: existence and algorithm analysis," IEEE Transactions on Signal Processing, vol. 56, no. 10, pp. 4563-4573, 2008.

[41] E. J. Kelly, "An adaptive detection algorithm," IEEE Transactions on Aerospace and Electronic Systems, vol. AES-22, no. 2, pp. 115-127, 1986.

[42] C. D. Richmond, "A note on non-Gaussian adaptive array detection and signal parameter estimation," IEEE Signal Processing Letters, vol. 3, no. 8, pp. 251-252, 1996.

[43] M. Jager, M. Neumann, S. Guillaso, and A. Reigber, "A self-initializing PolInSAR classifier using interferometric phase differences," IEEE Transactions on Geoscience and Remote Sensing, vol. 45, no. 11, pp. 3503-3518, 2007.

[44] G. Vasile, "Imagerie radar à synthse d'ouverture interférométrique et polarimétrique. Application au suivi des glaciers alpins," Ph.D. dissertation, Université de Savoie, France, 2007.

[45] G. Vasile, J. P. Ovarlez, F. Pascal, C. Tison, L. Bombrun, M. Gay, and E. Trouvé, "Normalized coherency matrix estimation under the SIRV model. Alpine glacier POLSAR data analysis," in Proceedings of the IEEE International Geoscience and Remote Sensing Symposium, Boston, USA, vol. 1, 2008, pp. I74-I77.

[46] S. R. Cloude and K. P. Papathanassiou, "Polarimetric SAR interferometry," IEEE Transactions on Geoscience and Remote Sensing, vol. 36, no. 5, pp. $1551-1565,1998$

[47] P. R. Kersten, J. S. Lee, and T. L. Ainsworth, "Unsupervised classification of polarimetric synthetic aperture radar images using fuzzy clustering and EM clustering," IEEE Transactions on Geoscience and Remote Sensing, vol. 43, no. 3, pp. 519-527, 2005.

[48] F. Cao, W. Hong, Y. Wu, and E. Pottier, "An unsupervised segmentation with an adaptive number of clusters using the SPAN/H/ $\alpha / A$ space and the complex Wishart clustering for fully polarimetric SAR data analysis," IEEE Transactions on Geoscience and Remote Sensing, vol. 45, no. 11, pp. 3454-3467, 2007.

[49] A. P. Doulgeris, S. N. Anfinsen, and T. Eltoft, "Classification with a non-Gaussian model for PolSAR data," IEEE Transactions on Geoscience and Remote Sensing, vol. 46, no. 10, pp. 2999-3009, 2008.

[50] E. L. Lehmann, Testing statistical hypotheses, 2nd ed., John-Wiley\&Sons, Ed. Springer-Verlag, New York, USA, 1986.

[51] P. Dreuillet, H. Cantalloube, E. Colin, P. Dubois-Fernandez, X. Dupuis, P. Fromage, F. Garestier, D. Heuze, H. Oriot, J. L. Peron, J. Peyret, G. Bonin, O. R. duPlessis, J. F. Nouvel, and B. Vaizan, "The ONERA RAMSES SAR: latest significant results and future developments," in Proceedings of the IEEE International Radar Conference, Verona, USA, 2006.

[52] J. S. Lee, S. R. Cloude, K. P. Papathanassiou, M. R. Grunes, and I. H. Woodhouse, "Speckle filtering and coherence estimation of polarimetric SAR interferometry data for forest applications," IEEE Transactions on Geoscience and Remote Sensing, vol. 41, no. 10, pp. 2254-2263, 2003.

[53] P. Imbo and J. C. Souyris, "Visualization of the polarimetric information: comparison betweenpartial versus full polarimetry architectures," in Proceedings of the IEEE International Geoscience and Remote Sensing Symposium, Honolulu, Hawaii, vol. 3, 2000, pp. 1116-1118.

[54] L. Ferro-Famil, E. Pottier, and J. S. Lee, "Classification and interpretation of polarimetric interferometric SAR data," in Proceedings of the IEEE International Geoscience and Remote Sensing Symposium, Toronto, Canada, vol. 1, 2002, pp. $635-637$.

[55] E. Pottier, L. Ferro-Famil, S. Allain, S. Cloude, I. Hajnsek, K. Papathanassiou, A. Moreira, M. Williams, T. Pearson, and Y. Desnos, "An overview of the PolSARpro v2.0 software. The educational toolbox for polarimetric and interferometric polarimetric SAR data processing," in POLinSAR 2007 Workshop, ESA, Franscati, Italy, 2007, pp. CD-ROM.

[56] J. M. Beaulieu and R. Touzi, "Segmentation of textured polarimetric SAR scenes by likelihood approximation," IEEE Transactions on Geoscience and Remote Sensing, vol. 42, no. 10, pp. 2063-2072, 2004.

[57] X. Zhang, L. Jiao, F. Liu, L. Bo, and M. Gong, "Spectral clustering ensemble applied to SAR image segmentation," IEEE Transactions on Geoscience and Remote Sensing, vol. 46, no. 7, pp. 2126-2136, 2008.

[58] S. Fukuda and H. Hirosawa, "Support vector machine classification of land cover: application to polarimetric SAR data," in Proceedings of the IEEE International Geoscience and Remote Sensing Symposium, Sydney, Australia, vol. 1, 2001, pp. 187-189.

[59] O. D'Hondt, L. Ferro-Famil, and E. Pottier, "The gradient structure tensor as an efficient descriptor of spatial texture in polarimetric SAR data," in Proceedings of the IEEE International Geoscience and Remote Sensing Symposium, Denver, USA, vol. 1, 2006, pp. 164-167.

[60] T. J. Barnard and D. D. Weiner, "Non-Gaussian clutter modeling with generalized spherically invariant random vectors," IEEE Transactions on Signal Processing, vol. 44, no. 10, pp. 2384-2390, 1996.

[61] O. D'Hondt, C. López-Martnez, L. Ferro-Famil, and E. Pottier, "Spatially nonstationary anisotropic texture analysis in SAR images," IEEE Transactions on Geoscience and Remote Sensing, vol. 45, no. 12, pp. 3905-3918, 2007. 


\section{LIST OF FIGURES}

The proposed estimation scheme. . . . . . . . . . . . . . . . . . . . . .

Simulated POLSAR data, Gaussian case $(200 \times 200$ pixels): (a) texture image, (b) initial 1-look span estimated using Eq. 3 and (c) amplitude color composition of the target vector elements $k_{1}-k_{3}-k_{2}$. . . . . . . . . . . . . .

Simulated POLSAR data, Gaussian case $(200 \times 200$ pixels $)$. Square root of LLMMSE span image using the normalized coherency estimated by: (a) BN-SCM, (b) BN-FP and (c) SDAN-FP. Color composition of the normalized coherency diagonal elements $[M]_{11-}[M]_{33}-[M]_{22}$ estimated by: (d) BN-SCM, (e) BN-FP and (f) SDAN-FP. . . .

4 Simulated POLSAR data, SIRV case $(200 \times 200$ pixels); (a) texture image, (b) initial 1-look span estimated using Eq. 3 and (c) amplitude color composition of the target vector elements $k_{1}-k_{3}-k_{2}$. . . . . . . . . . . . . . .

5 Simulated POLSAR data, SIRV case $(200 \times 200$ pixels). Square root of LLMMSE span image using the normalized coherency estimated by: (a) BN-SCM, (b) BN-FP and (c) SDAN-FP. Color composition of the normalized coherency diagonal elements $[M]_{11}-[M]_{33}-[M]_{22}$ estimated by: (d) BN-SCM, (e) BN-FP and (f) SDAN-FP. . . .

Brétigny, RAMSES POLSAR data, X-band ( $501 \times 501$ pixels): (a) amplitude color composition of the target vector elements $k_{1}-k_{3}-k_{2}$ and (b) optical image $(137 \times 137$ pixel zoom of the initial span superposed for illustrating the region of interest). . . . . . . . . . . . . . . . . . . . . . . . . . .

Brétigny, RAMSES POLSAR data, X-band $(137 \times 137$ pixels). Square root of LLMMSE span image using the normalized coherency estimated by: (a) BN-SCM, (b) BN-FP and (c) SDAN-FP. Color composition of the normalized coherency diagonal elements $[M]_{11-}[M]_{33}-[M]_{22}$ estimated by: (d) BN-SCM, (e) BN-FP and (f) SDAN-FP. Color composition of the polarimetric $H-\alpha$ - $A$ parameters estimated by: (g) BN-SCM, (h) BN-FP and (i) SDAN-FP. . . . . . . . . . . . . . . . . . . . . . . . . . . . . . . .

Brétigny, RAMSES POLSAR data, X-band ( $137 \times 137$ pixels). Color composition of the coherency diagonal elements $[T]_{11-}[T]_{33}-[T]_{22}$ estimated by: (d) BN, (e) IDAN and (f) SDAN-FP after multiplication with the LLMMSE span from Fig. 7-(c). $H$ - $\alpha$ classification results using: (d) BN, (e) IDAN and (f) SDAN-FP. . . . . . . . . . . . .

Brétigny, RAMSES POLSAR data, X-band (501 × 501 pixels). LLMMSE span using the normalized coherency estimated by SDAN-FP: (a) span image, (d) Gamma unsupervised classification and (g) physical mechanism identification (odd bounce classes, even bounce classes, volume class) using the SDAN-FP normalized coherency. SDAN-FP coherency matrix after span multiplication: (b) color composition of the diagonal elements $[T]_{11^{-}}[T]_{33^{-}}$ $[T]_{22}$, (e) Wishart unsupervised classification and (h) physical mechanism identification. SDAN-FP normalized coherency matrix: (c) color composition of the diagonal elements $[M]_{11}-[M]_{33}-[M]_{22}$, (f) SIRV unsupervised classification and (i) physical mechanism identification. . . . . . . . . . . . . . . . . . . . . . .

10 Toulouse, RAMSES POLSAR data, X-band $(500 \times 500$ pixels), resolution azimuth and range of $50 \mathrm{~cm}$ : (a) amplitude color composition of the target vector elements $k_{1}-k_{3}-k_{2}$ and (b) optical image (C) NES/Spot-Image. .

11 Toulouse, RAMSES POLSAR data, X-band $(500 \times 500$ pixels), resolution azimuth and range of $50 \mathrm{~cm}$. Color composition of the diagonal elements of the diagonal elements $[T]_{11}-[T]_{33}-[T]_{22}$ : (a) the Lee refined filter, (b) SDAN-FP coherency matrix after span multiplication. (c) color composition of the diagonal elements $[M]_{11}$ $[M]_{33}-[M]_{22}$ estimated by SDAN-FP. Wishart unsupervised classification: (d) coherency estimated by the Lee refined filter, (e) SDAN-FP coherency matrix after span multiplication. (f) unsupervised classification of the SDAN-FP normalized coherency based on the SIRV distance measure. Physical mechanism identification (odd bounce classes, even bounce classes, volume class) using: ( $\mathrm{g}$ ) coherency estimated by the Lee refined filter, (h) SDAN-FP coherency matrix after span multiplication, (i) SDAN-FP normalized coherency. . . . . . . . . . . . 


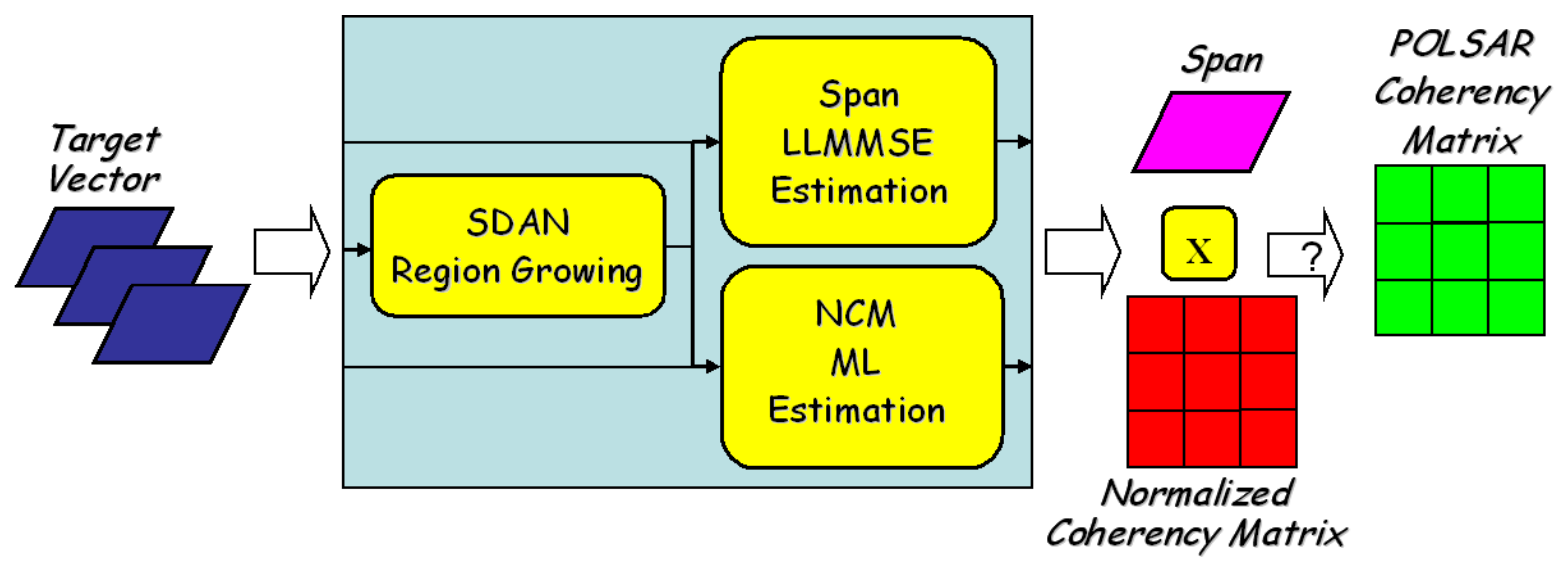

Fig. 1. The proposed estimation scheme. 


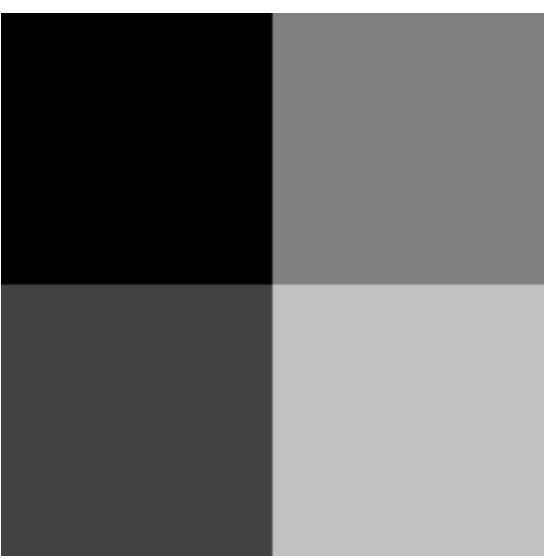

(a)

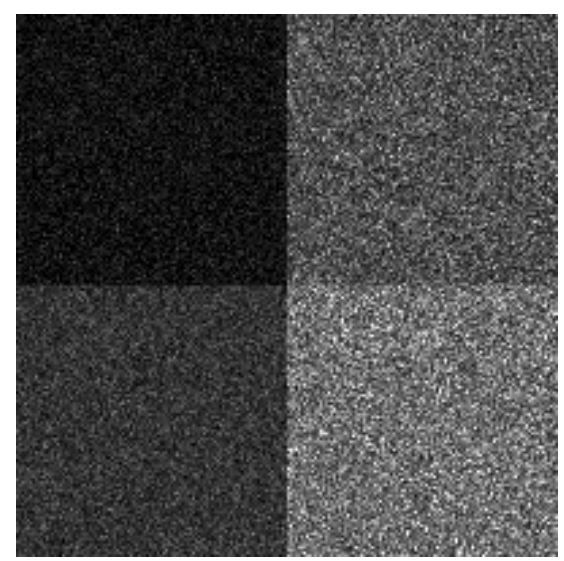

(b)

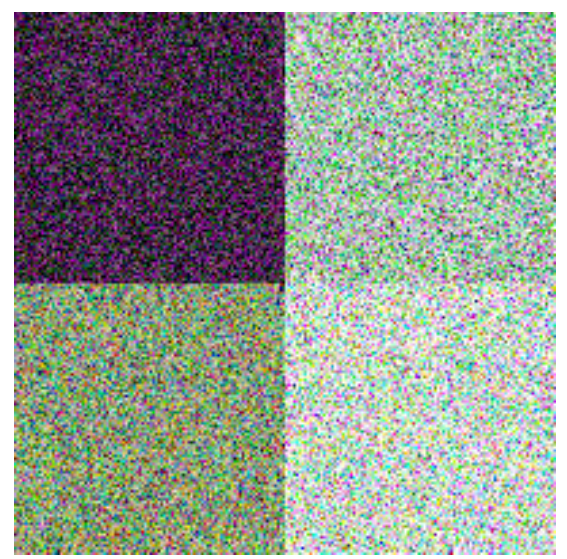

(c)

Fig. 2. Simulated POLSAR data, Gaussian case $(200 \times 200$ pixels): (a) texture image, (b) initial 1-look span estimated using Eq. 3 and (c) amplitude color composition of the target vector elements $k_{1}-k_{3}-k_{2}$. 


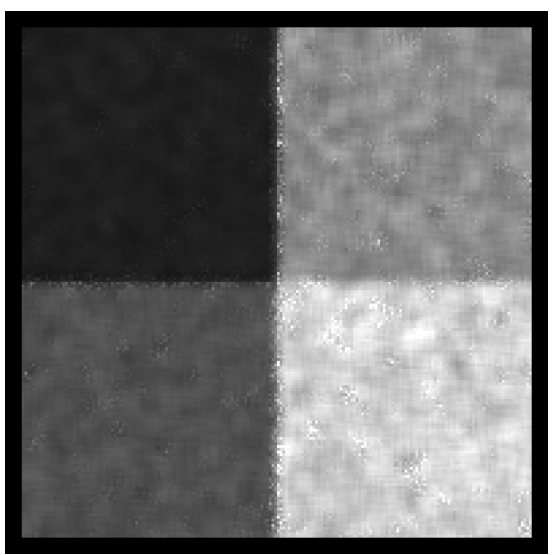

(a)

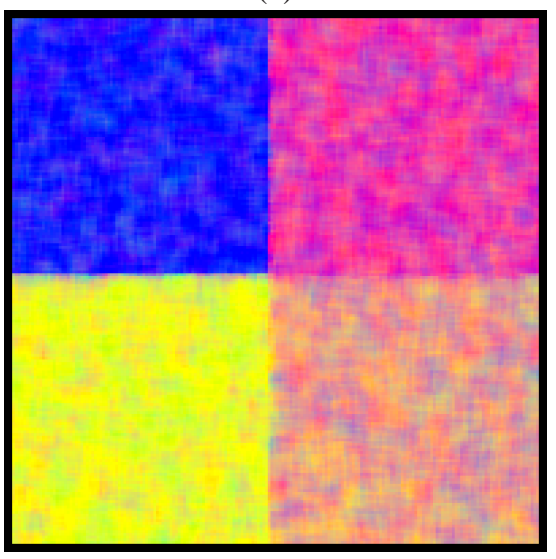

(d)

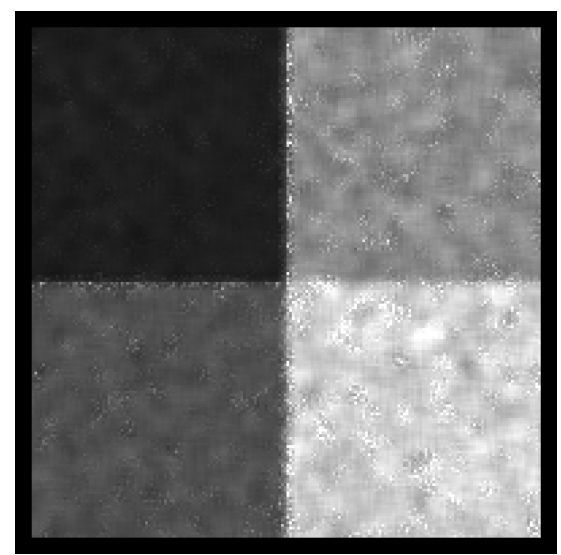

(b)

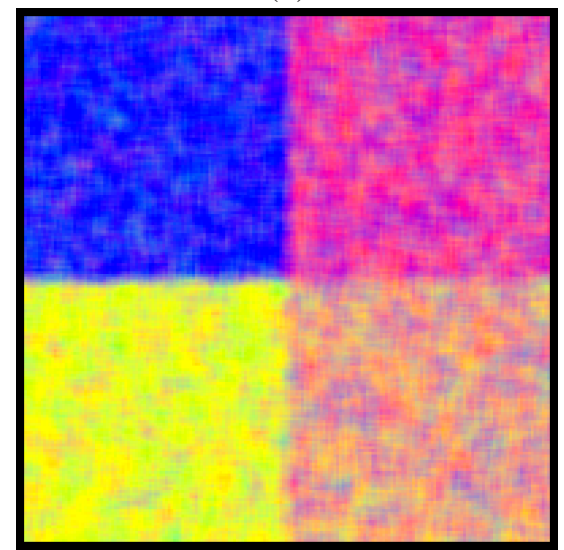

(e)

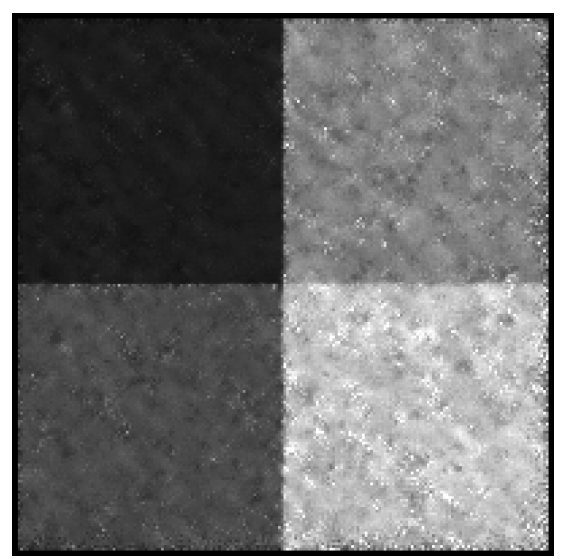

(c)

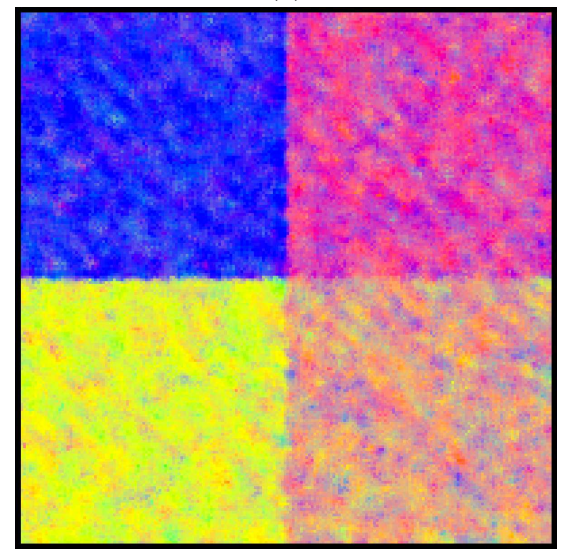

(f)

Fig. 3. Simulated POLSAR data, Gaussian case $(200 \times 200$ pixels). Square root of LLMMSE span image using the normalized coherency estimated by: (a) BN-SCM, (b) BN-FP and (c) SDAN-FP. Color composition of the normalized coherency diagonal elements $[M]_{11}-[M]_{33}-[M]_{22}$ estimated by: (d) BN-SCM, (e) BN-FP and (f) SDAN-FP. 


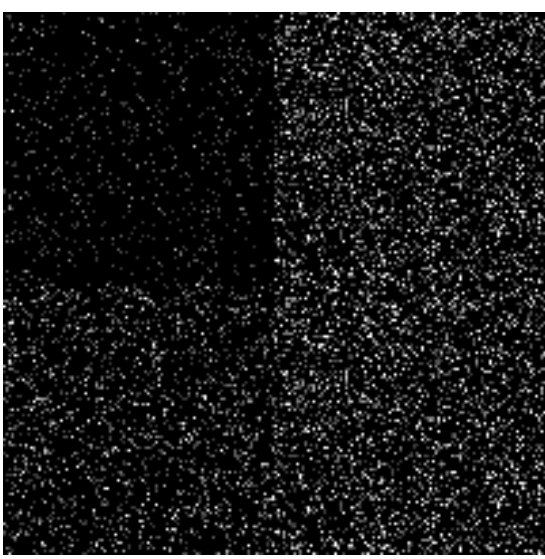

(a)

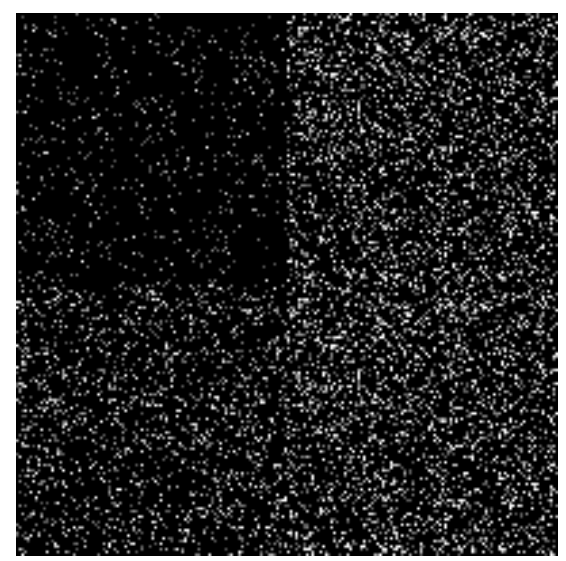

(b)

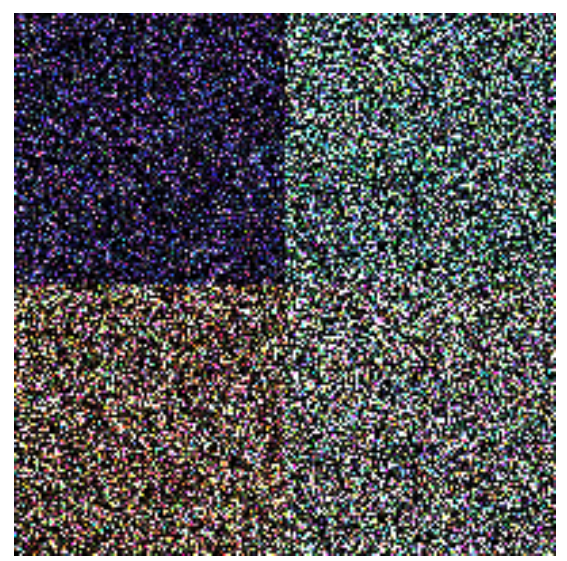

(c)

Fig. 4. Simulated POLSAR data, SIRV case $(200 \times 200$ pixels); (a) texture image, (b) initial 1-look span estimated using Eq. 3 and (c) amplitude color composition of the target vector elements $k_{1}-k_{3}-k_{2}$. 


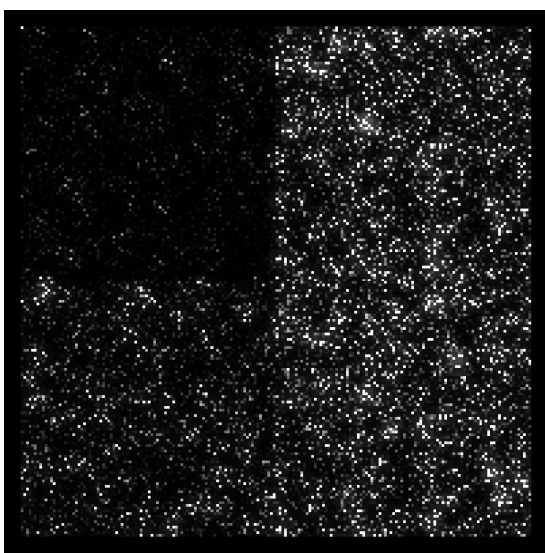

(a)

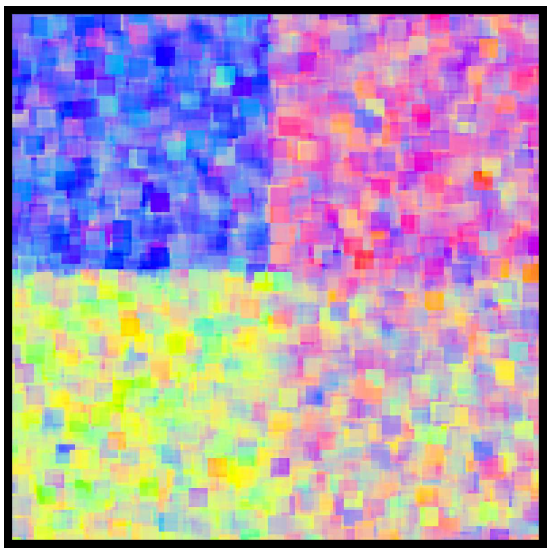

(d)

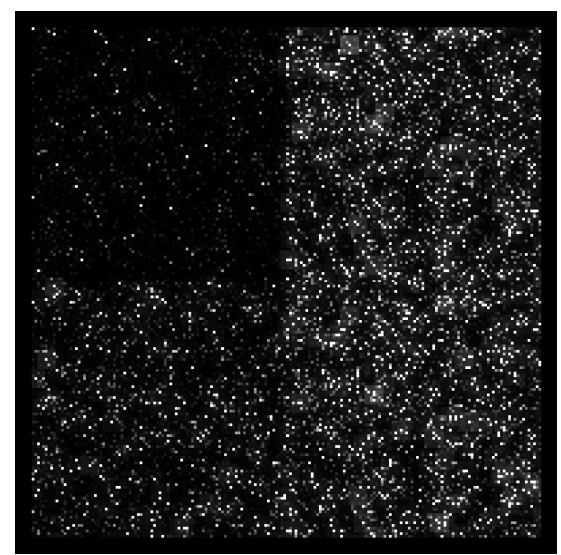

(b)

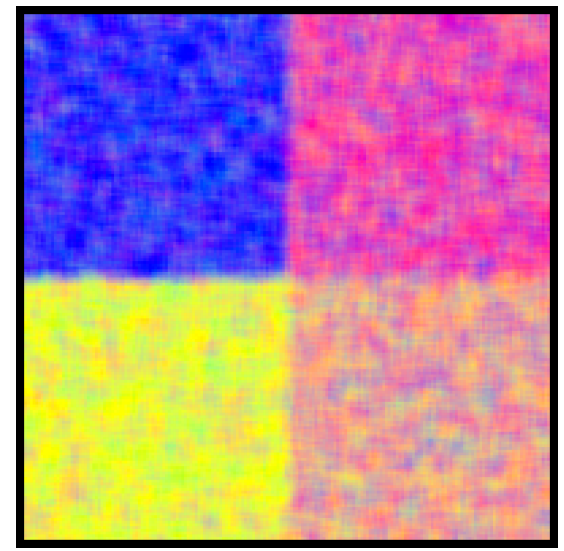

(e)

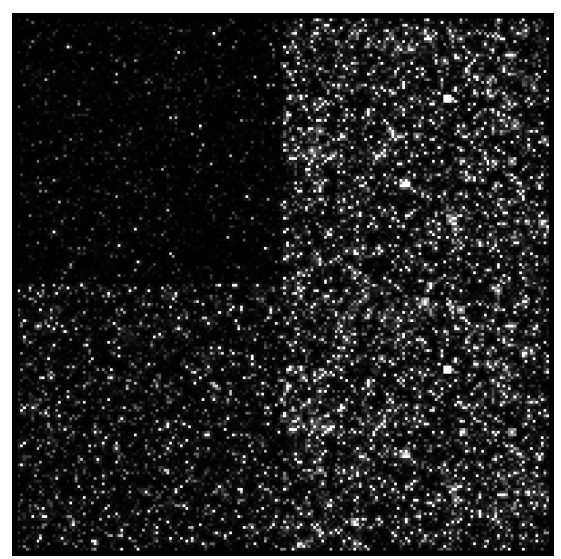

(c)

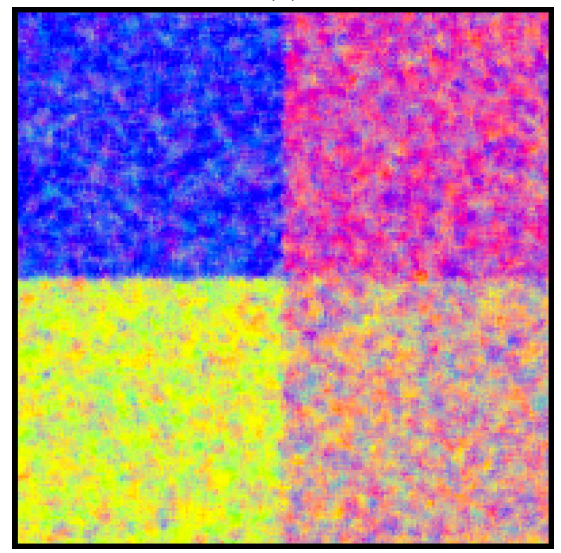

(f)

Fig. 5. Simulated POLSAR data, SIRV case $(200 \times 200$ pixels). Square root of LLMMSE span image using the normalized coherency estimated by: (a) BN-SCM, (b) BN-FP and (c) SDAN-FP. Color composition of the normalized coherency diagonal elements $[M]_{11}-[M]_{33}-[M]_{22}$ estimated by: (d) BN-SCM, (e) BN-FP and (f) SDAN-FP. 


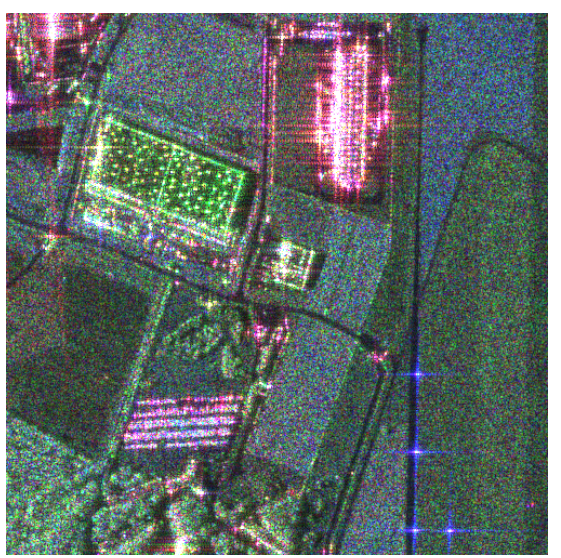

(a)

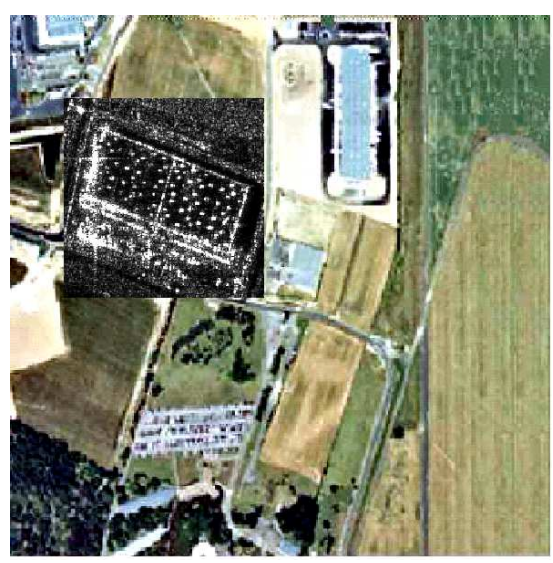

(b)

Fig. 6. Brétigny, RAMSES POLSAR data, X-band ( $501 \times 501$ pixels): (a) amplitude color composition of the target vector elements $k_{1}-k_{3}-k_{2}$ and (b) optical image $(137 \times 137$ pixel zoom of the initial span superposed for illustrating the region of interest). 


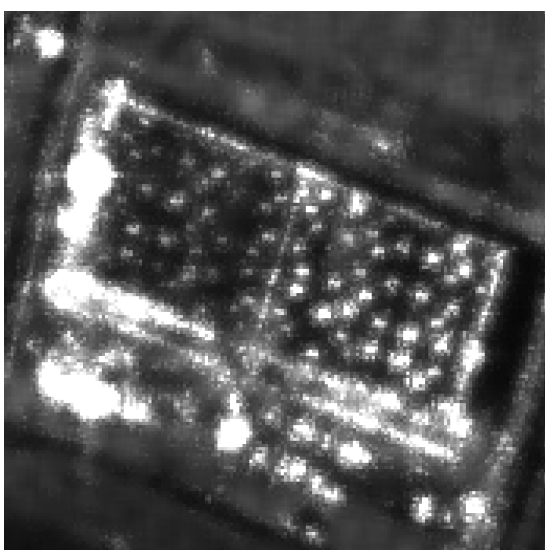

(a)

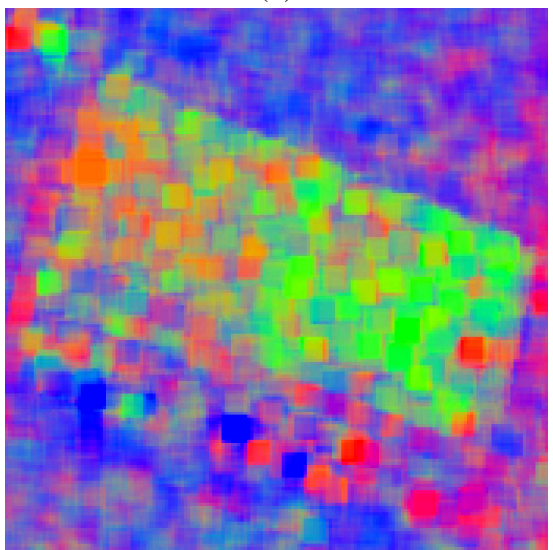

(d)

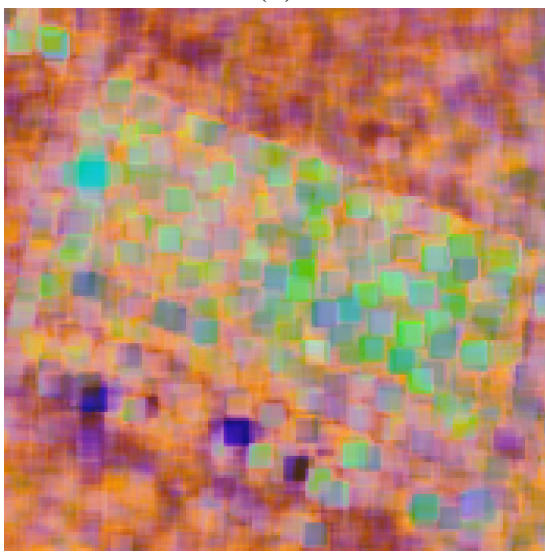

(g)

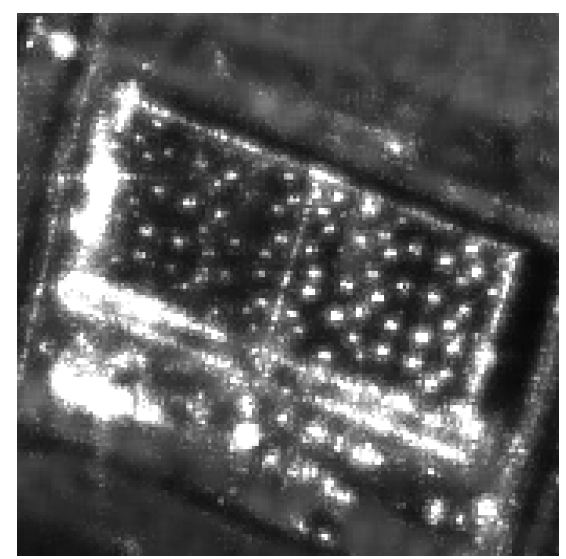

(b)

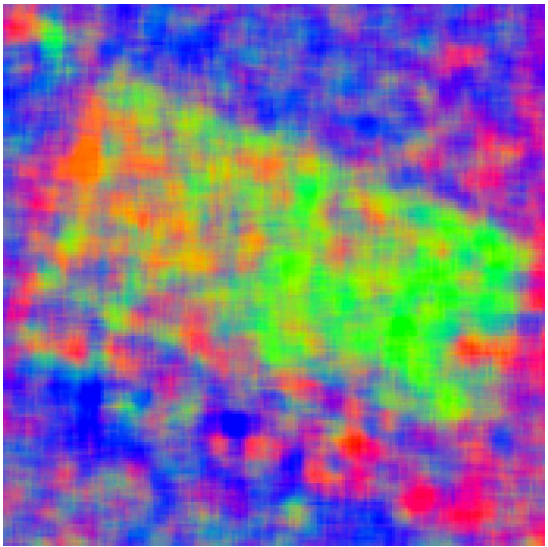

(e)

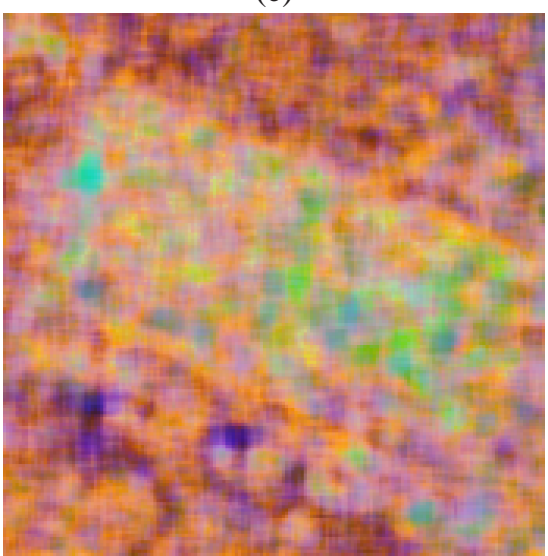

(h)

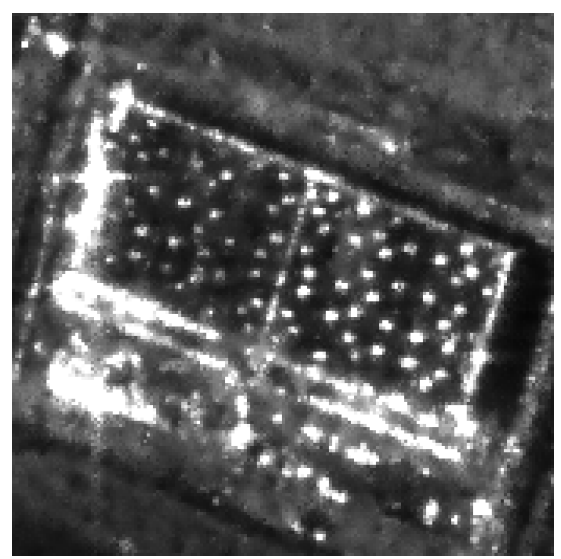

(c)

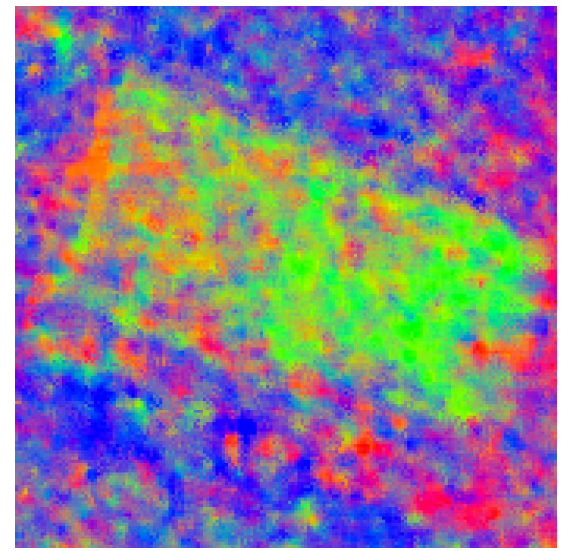

(f)

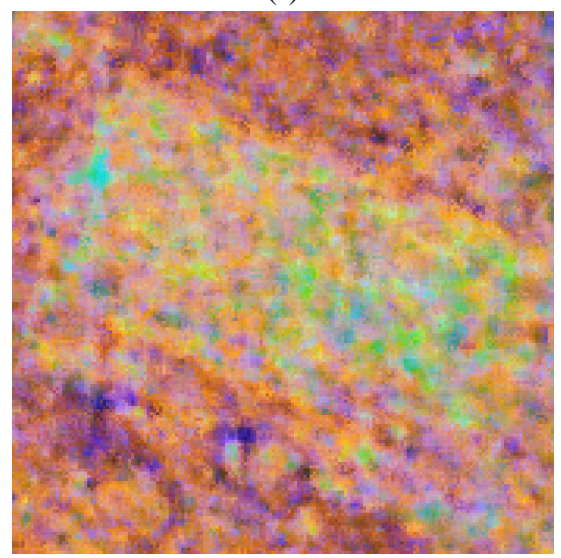

(i)

Fig. 7. Brétigny, RAMSES POLSAR data, X-band $(137 \times 137$ pixels). Square root of LLMMSE span image using the normalized coherency estimated by: (a) BN-SCM, (b) BN-FP and (c) SDAN-FP. Color composition of the normalized coherency diagonal elements $[M]_{11}-[M]_{33}-[M]_{22}$ estimated by: (d) BN-SCM, (e) BN-FP and (f) SDAN-FP. Color composition of the polarimetric $H$ - $\alpha$ - $A$ parameters estimated by: (g) BN-SCM, (h) BN-FP and (i) SDAN-FP. 


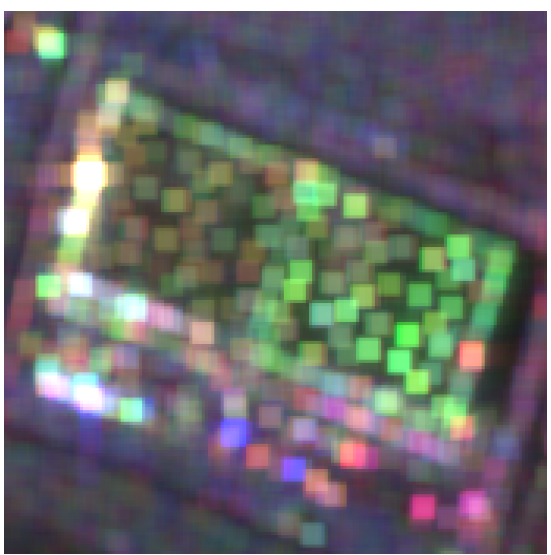

(a)

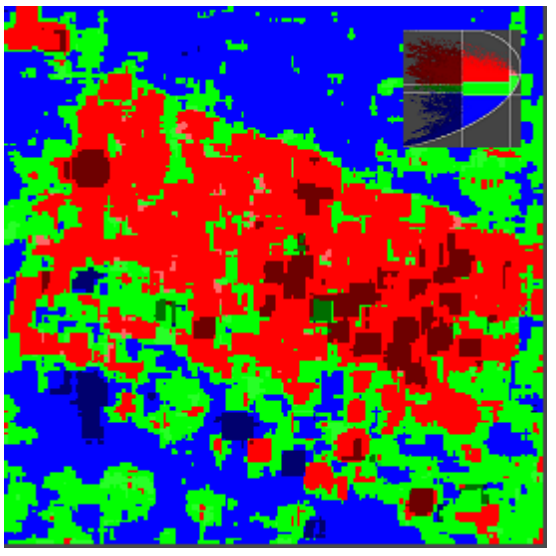

(d)

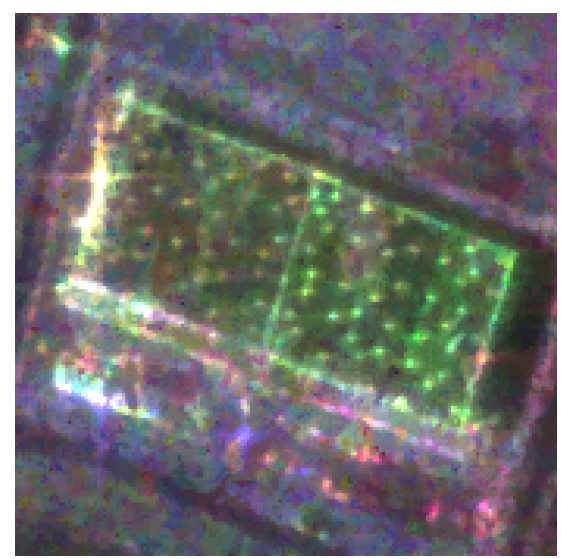

(b)

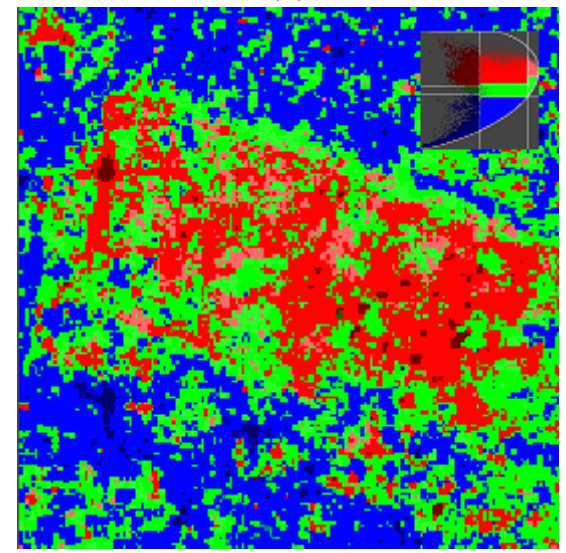

(e)

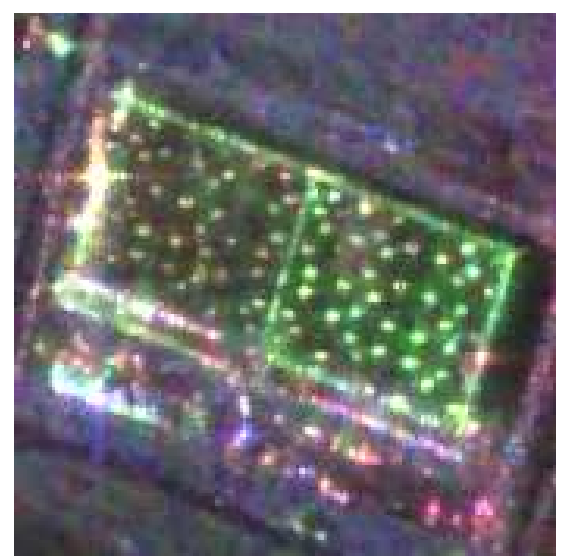

(c)

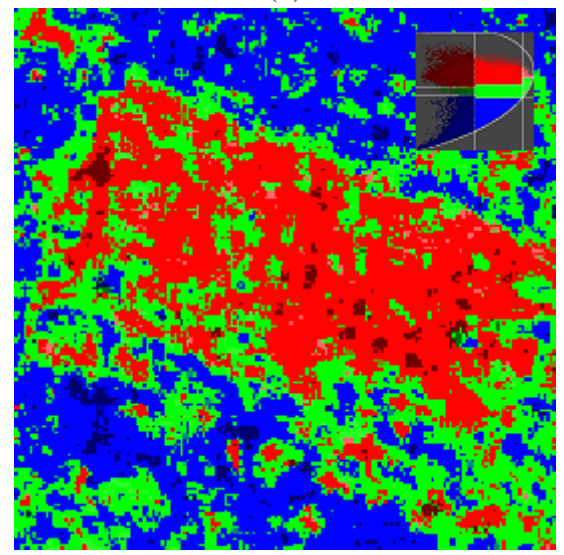

(f)

Fig. 8. Brétigny, RAMSES POLSAR data, X-band $\left(137 \times 137\right.$ pixels). Color composition of the coherency diagonal elements $[T]_{11}-[T]_{33}-[T]_{22}$ estimated by: (d) BN, (e) IDAN and (f) SDAN-FP after multiplication with the LLMMSE span from Fig. 7-(c). $H$ - $\alpha$ classification results using: (d) BN, (e) IDAN and (f) SDAN-FP. 


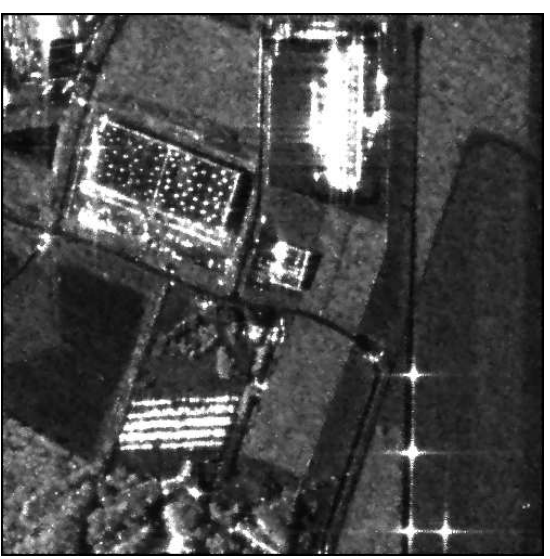

(a)

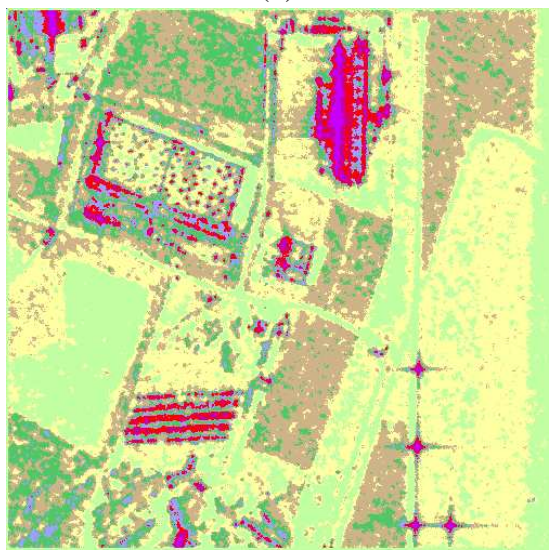

(d)

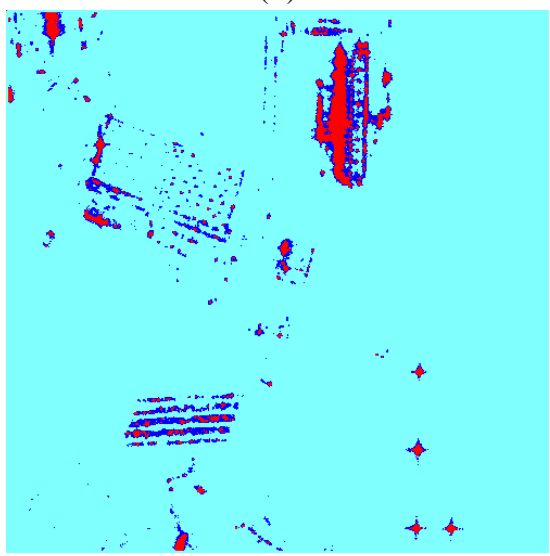

(g)

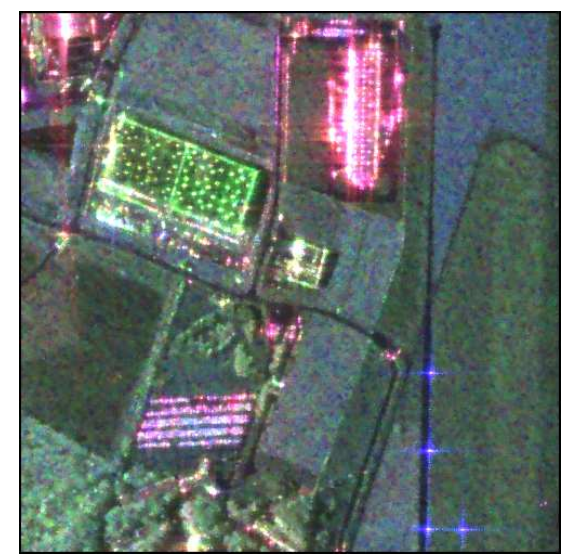

(b)

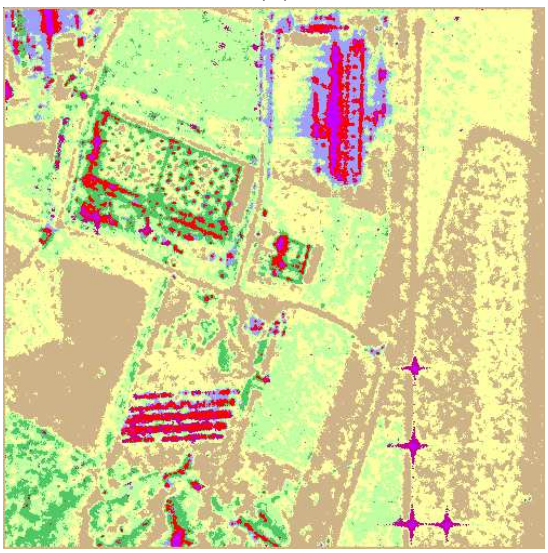

(e)

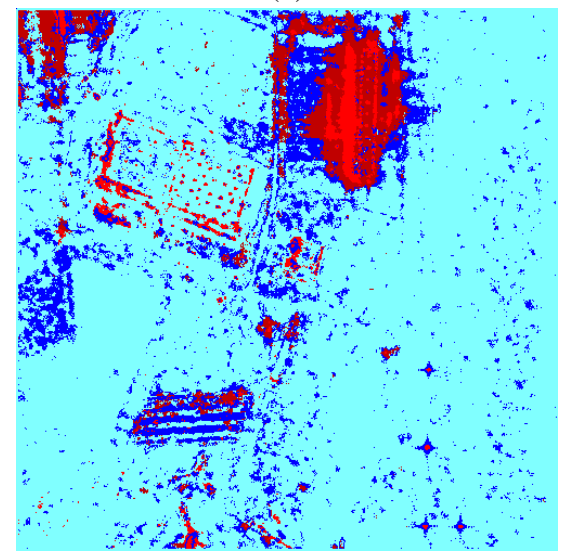

(h)

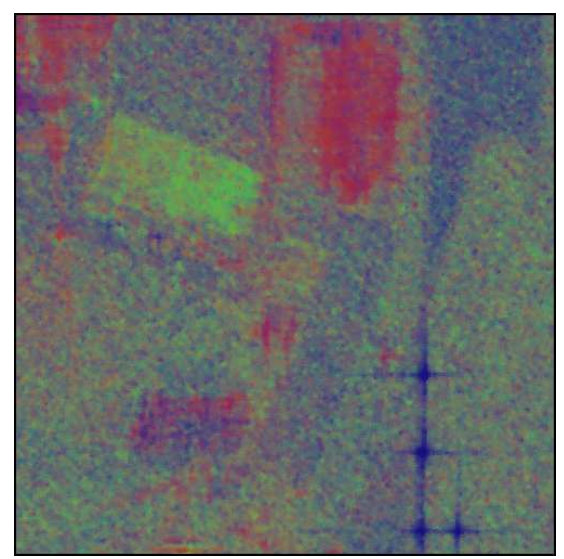

(c)

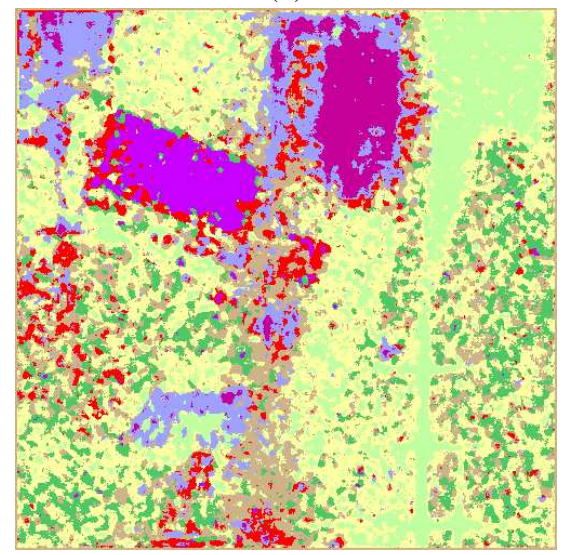

(f)

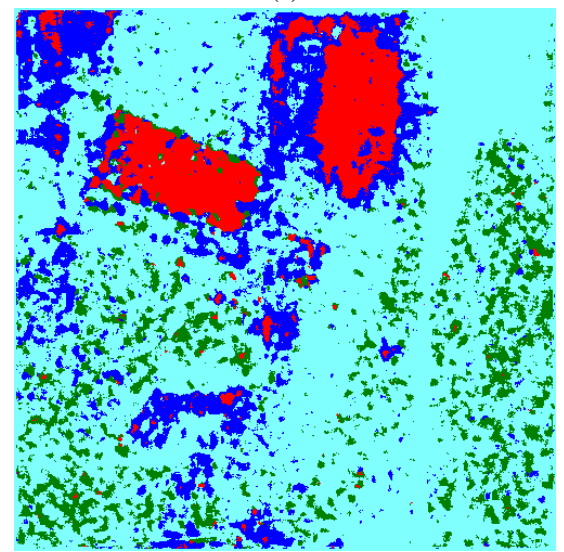

(i)

Fig. 9. Brétigny, RAMSES POLSAR data, X-band $(501 \times 501$ pixels). LLMMSE span using the normalized coherency estimated by SDAN-FP: (a) span image, (d) Gamma unsupervised classification and (g) physical mechanism identification (odd bounce classes, even bounce classes, volume class) using the SDAN-FP normalized coherency. SDAN-FP coherency matrix after span multiplication: (b) color composition of the diagonal elements $\left.[T]_{11-}-\right]_{33}-[T]_{22},(\mathrm{e})$ Wishart unsupervised classification and (h) physical mechanism identification. SDAN-FP normalized coherency matrix: (c) color composition of the diagonal elements $[M]_{11}-[M]_{33}-[M]_{22}$, (f) SIRV unsupervised classification and (i) physical mechanism identification. 


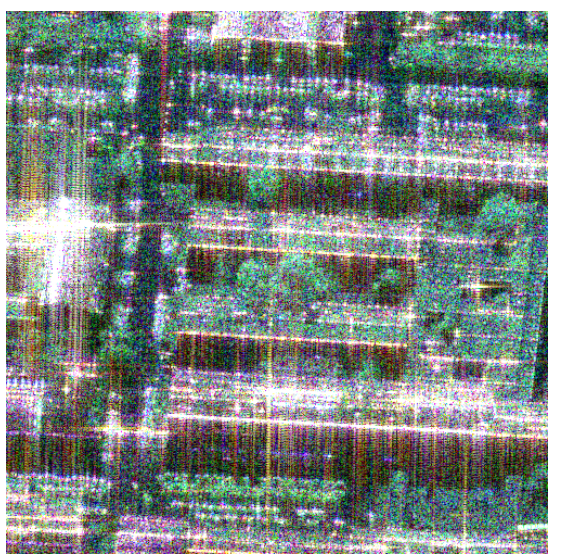

(a)

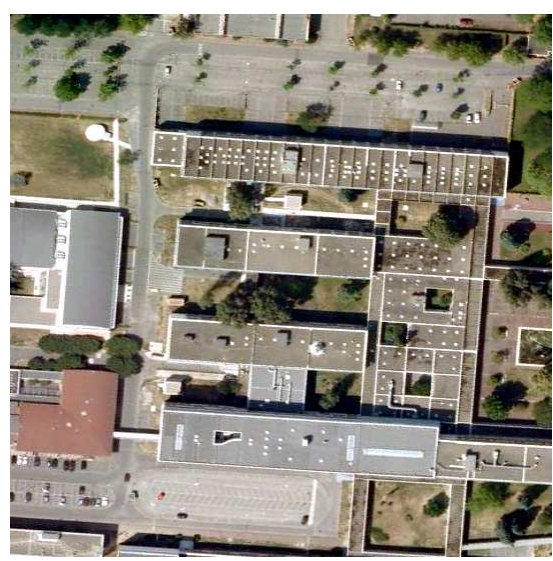

(b)

Fig. 10. Toulouse, RAMSES POLSAR data, X-band $(500 \times 500$ pixels), resolution azimuth and range of $50 \mathrm{~cm}$ : (a) amplitude color composition of the target vector elements $k_{1}-k_{3}-k_{2}$ and (b) optical image (C) CNES/Spot-Image. 


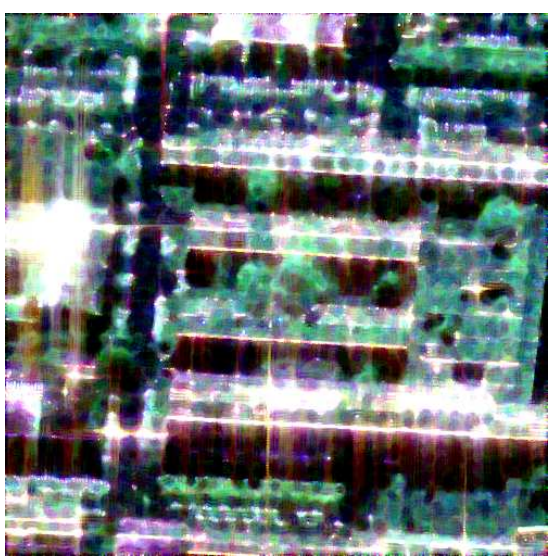

(a)

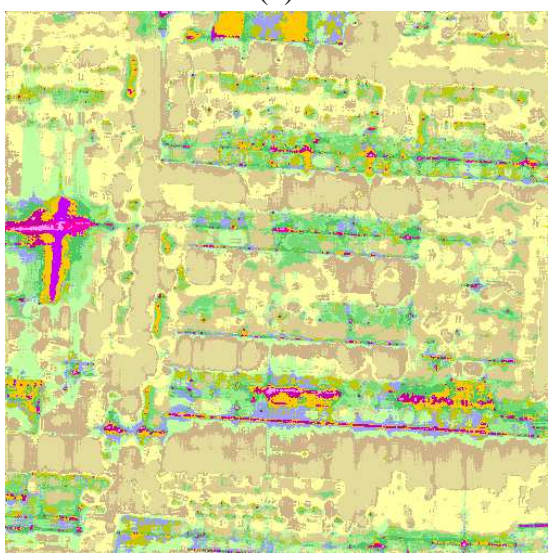

(d)

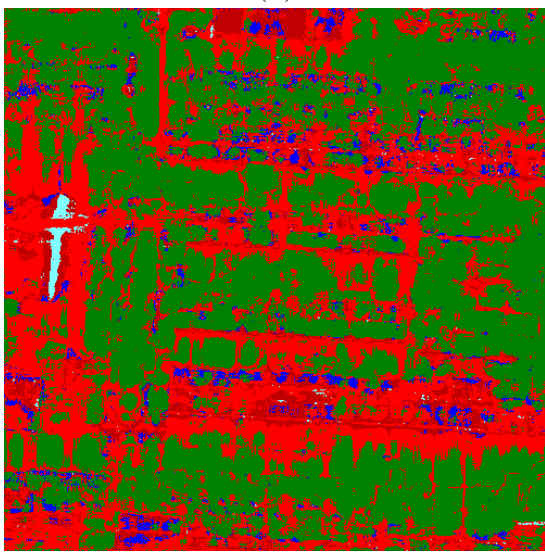

(g)

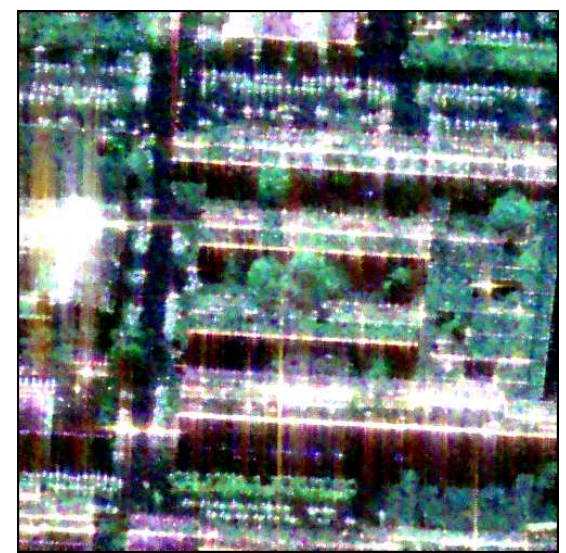

(b)

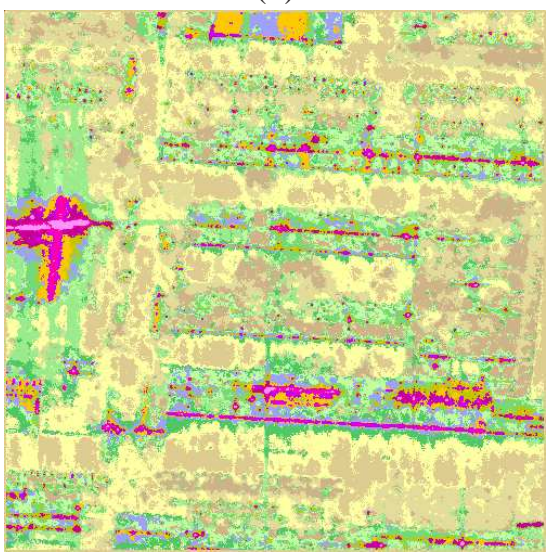

(e)

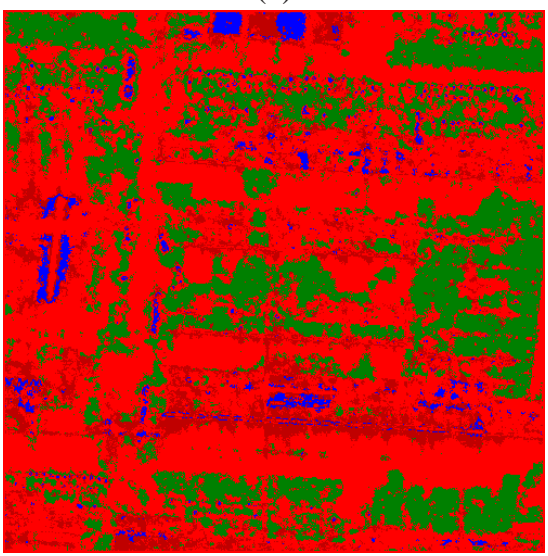

(h)

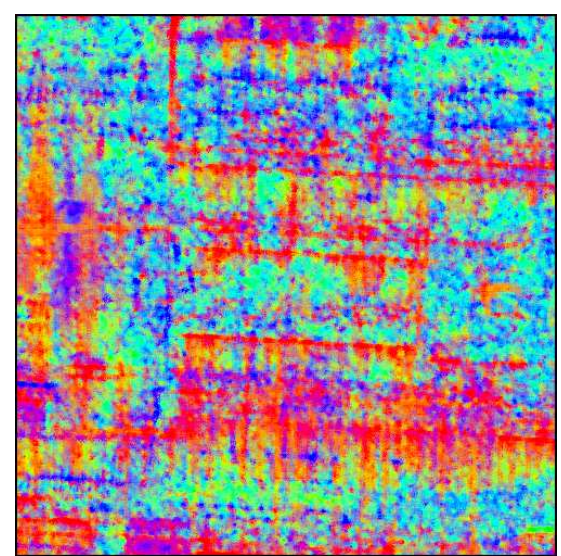

(c)

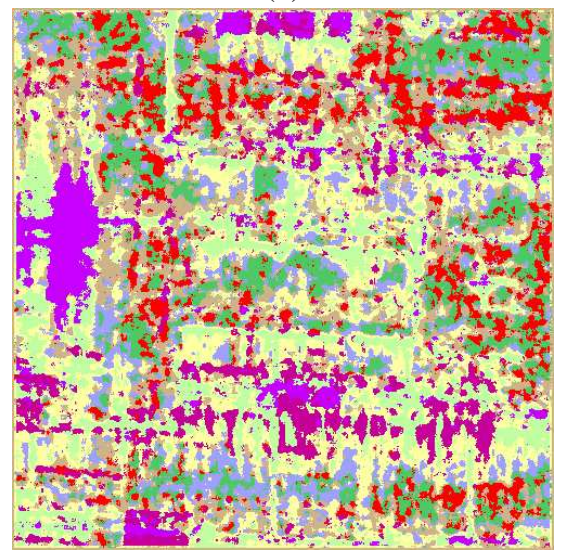

(f)

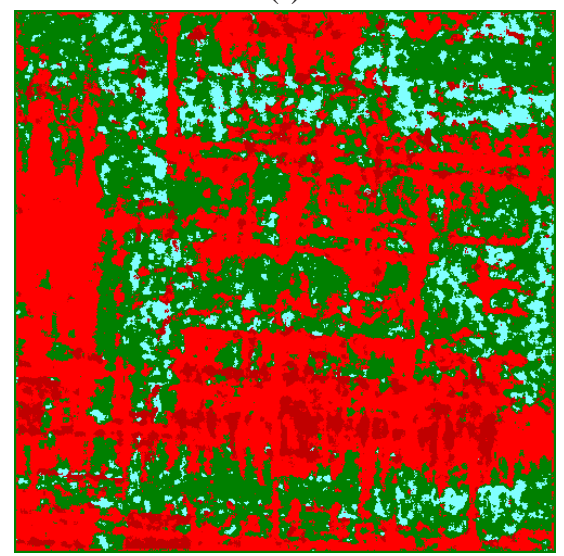

(i)

Fig. 11. Toulouse, RAMSES POLSAR data, X-band $(500 \times 500$ pixels $)$, resolution azimuth and range of $50 \mathrm{~cm}$. Color composition of the diagonal elements of the diagonal elements $[T]_{11}-[T]_{33}-[T]_{22}$ : (a) the Lee refined filter, (b) SDAN-FP coherency matrix after span multiplication. (c) color composition of the diagonal elements $[M]_{11}-[M]_{33}-[M]_{22}$ estimated by SDAN-FP. Wishart unsupervised classification: (d) coherency estimated by the Lee refined filter, (e) SDAN-FP coherency matrix after span multiplication. (f) unsupervised classification of the SDAN-FP normalized coherency based on the SIRV distance measure. Physical mechanism identification (odd bounce classes, even bounce classes, volume class) using: (g) coherency estimated by the Lee refined filter, (h) SDAN-FP coherency matrix after span multiplication, (i) SDAN-FP normalized coherency. 


\section{LIST OF TABLES}

I Simulated POLSAR data, Gaussian case: mean and standard deviation of normalized coherency elements over

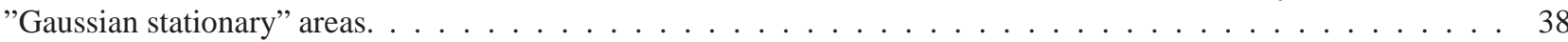

II Simulated POLSAR data, Gaussian case: span mean ratio $\widehat{\mu} / \mu_{\text {ref }}$ and coefficient of variation over "Gaussian stationary" areas. . . . . . . . . . . . . . . . . . . . . . . . . 39

III Simulated POLSAR data, SIRV case: mean and standard deviation of normalized coherency elements over "SIRP

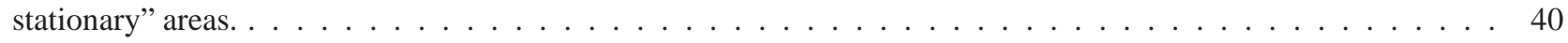

IV Simulated POLSAR data, SIRV case: the Kolmogorov-Smirnov test $K S_{n}=\max _{x}\left|F_{n}(x)-F_{r e f}(x)\right|$ for the

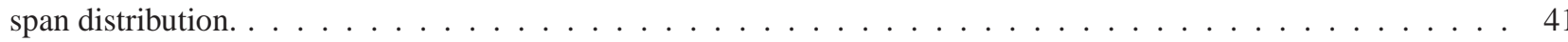


TABLE I

Simulated POLSAR DATA, GAussian CASE: MEAN AND STANDARD DEVIATION OF NORMALIZED COHERENCY ELEMENTS OVER "GAUSSIAN STATIONARY" AREAS.

\begin{tabular}{|c|c||c|c|c||c|c|c|}
\hline \multirow{2}{*}{$\begin{array}{c}\text { Parameter } \\
(3378 \text { pixels })\end{array}$} & \multirow{2}{*}{ Value } & \multicolumn{3}{|c||}{ Mean } & \multicolumn{3}{c|}{ Standard deviation } \\
\cline { 3 - 8 } & & BN-SCM & BN-FP & SDAN-FP & BN-SCM & BN-FP & SDAN-FP \\
\hline \hline$M_{11}$ & 1.79 & 1.78 & 1.77 & 1.77 & 0.13 & 0.15 & 0.19 \\
\hline$M_{22}$ & 0.77 & 0.78 & 0.78 & 0.78 & 0.11 & 0.12 & 0.15 \\
\hline$M_{33}$ & 0.43 & 0.44 & 0.44 & 0.44 & 0.06 & 0.07 & 0.09 \\
\hline$\Re\left\{M_{12}\right\}$ & 0.01 & 0.01 & 0.01 & 0.01 & 0.10 & 0.13 & 0.16 \\
\hline$\Im\left\{M_{12}\right\}$ & -0.19 & -0.17 & -0.17 & -0.17 & 0.12 & 0.13 & 0.16 \\
\hline$\Re\left\{M_{13}\right\}$ & 0.07 & 0.08 & 0.08 & 0.08 & 0.09 & 0.10 & 0.11 \\
\hline$\Im\left\{M_{13}\right\}$ & 0.03 & 0.03 & 0.03 & 0.03 & 0.09 & 0.10 & 0.12 \\
\hline$\Re\left\{M_{23}\right\}$ & 0.16 & 0.15 & 0.15 & 0.15 & 0.06 & 0.07 & 0.08 \\
\hline$\Im\left\{M_{23}\right\}$ & 0.02 & 0.02 & 0.02 & 0.02 & 0.06 & 0.07 & 0.08 \\
\hline Mean normalized error $(\epsilon)$ & 0.17 & 0.19 & 0.23 & & & \\
\hline
\end{tabular}


TABLE II

SimUlated POLSAR DATA, GAUSSIAN CASE: SPAN MEAN RATIO $\widehat{\mu} / \mu_{\text {ref }}$ AND COEFFICIENT OF VARIATION OVER "GaUSSIAN STATIONARY" AREAS.

\begin{tabular}{||c||c|c|c||c|c|c||}
\hline \hline \multirow{2}{*}{$\begin{array}{c}\text { Span } \\
\text { (3378 pixels) }\end{array}$} & \multicolumn{4}{c||}{ Mean ratio } & \multicolumn{3}{c||}{ Coefficient of variation } \\
\cline { 2 - 7 } & BN-SCM & BN-FP & SDAN-FP & BN-SCM & BN-FP & SDAN-FP \\
\hline \hline LLMMSE & 0.99 & 1.07 & 0.99 & 0.10 & 0.13 & 0.16 \\
\hline \hline
\end{tabular}


TABLE III

SIMULATED POLSAR DATA, SIRV CASE: MEAN AND STANDARD DEVIATION OF NORMALIZED COHERENCY ELEMENTS OVER "SIRP STATIONARY" AREAS.

\begin{tabular}{|c|c||c|c|c||c|c|c|}
\hline \multirow{2}{*}{$\begin{array}{c}\text { Parameter } \\
(3378 \text { pixels })\end{array}$} & \multirow{2}{*}{ Value } & \multicolumn{3}{|c||}{ Mean } & \multicolumn{3}{c|}{ Standard deviation } \\
\cline { 3 - 8 } & & BN-SCM & BN-FP & SDAN-FP & BN-SCM & BN-FP & SDAN-FP \\
\hline \hline$M_{11}$ & 1.79 & 1.75 & 1.80 & 1.76 & 0.35 & 0.14 & 0.24 \\
\hline$M_{22}$ & 0.77 & 0.75 & 0.75 & 0.79 & 0.27 & 0.10 & 0.20 \\
\hline$M_{33}$ & 0.43 & 0.49 & 0.44 & 0.44 & 0.23 & 0.08 & 0.11 \\
\hline$\Re\left\{M_{12}\right\}$ & 0.01 & 0.04 & 0.01 & 0.02 & 0.36 & 0.14 & 0.22 \\
\hline$\Im\left\{M_{12}\right\}$ & -0.19 & -0.17 & -0.19 & -0.17 & 0.35 & 0.11 & 0.24 \\
\hline$\Re\left\{M_{13}\right\}$ & 0.07 & 0.09 & 0.07 & 0.06 & 0.30 & 0.11 & 0.17 \\
\hline$\Im\left\{M_{13}\right\}$ & 0.03 & 0.04 & 0.04 & 0.04 & 0.26 & 0.09 & 0.17 \\
\hline$\Re\left\{M_{23}\right\}$ & 0.16 & 0.18 & 0.16 & 0.15 & 0.18 & 0.06 & 0.10 \\
\hline$\Im\left\{M_{23}\right\}$ & 0.02 & 0.03 & 0.02 & 0.02 & 0.19 & 0.06 & 0.10 \\
\hline Mean normalized error $(\epsilon)$ & 0.51 & 0.19 & 0.28 & & & \\
\hline
\end{tabular}


TABLE IV

SimUlated POLSAR DATA, SIRV CASE: THE KOLMOGOROV-SMIRNOV TEST $K S_{n}=\max _{x}\left|F_{n}(x)-F_{r e f}(x)\right|$ FOR THE SPAN DISTRIBUTION.

\begin{tabular}{||c||c|c|c||}
\hline \hline \multirow{2}{*}{$\begin{array}{c}\text { Span } \\
\text { (3378 pixels) }\end{array}$} & \multicolumn{3}{c||}{ K-S test } \\
\cline { 2 - 4 } & BN-SCM & BN-FP & SDAN-FP \\
\hline \hline LLMMSE & 0.111 & 0.100 & 0.068 \\
\hline \hline
\end{tabular}

